ALEA, Lat. Am. J. Probab. Math. Stat. 18, 1529-1560 (2021)

DOI: $10.30757 /$ ALEA.v18-56

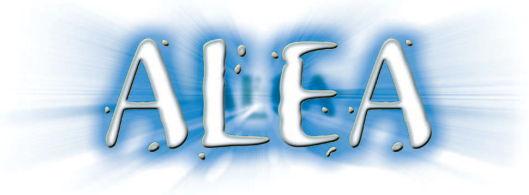

\title{
Stationary Harmonic Measure as the Scaling Limit of Truncated Harmonic Measure
}

\author{
Eviatar B. Procaccia, Jiayan Ye and Yuan Zhang \\ Texas A\&M University \& Technion - Israel Institute of Technology \\ College Station, TX, United States \& Haifa, Israel. \\ E-mail address: eviatarp@technion.ac.il \\ $U R L$ : https://sites.google.com/site/ebprocaccia \\ Ben-Gurion University of the Negev \\ Be'er Sheva, Israel. \\ E-mail address: yej@post.bgu.ac.il \\ URL: https://www.math.pku.edu.cn/teachers/zhangyuan/eindex.html \\ Peking University \\ Beijing, China. \\ E-mail address: zhangyuan@math.pku.edu.cn
}

\begin{abstract}
In this paper we prove that the stationary harmonic measure of an infinite set in the upper planar lattice can be represented as the proper scaling limit of the classical harmonic measure of truncations of the infinite set.
\end{abstract}

\section{Introduction}

Motivated by the study of Diffusion Limited Aggregation (DLA) on graphs with absorbing boundaries, recently Procaccia and Zhang $(2019,2021)$ an appropriate harmonic measure was defined on the upper planar lattice. The so-called stationary harmonic measure is a natural growth measure for DLA in the upper planar lattice. In Procaccia and Zhang (2019) a finite DLA process (i.e., DLA such that the size of initial configuration is finite) on the upper planar lattice was defined and studied. Moreover, an infinite stationary process that bounds from above any processes generated by the stationary harmonic measure was defined. However, the most interesting process we wish to study is an infinite stationary DLA (SDLA). The well-definedness of infinite SDLA is not straight-forward because the stationary harmonic measure is unbounded (see Procaccia et al., 2020 for details). In the subsequent paper Procaccia et al. (2020), we construct an infinite SDLA on the

Received by the editors June 12th, 2019; accepted May 9th, 2021.

2010 Mathematics Subject Classification. 60K35, 60G50.

Key words and phrases. Diffusion limited aggregation, harmonic measure, stationary diffusion limited aggregation, stationary harmonic measure.

E. B. Procaccia: Research supported by NSF grant DMS-1812009.

Y. Zhang: Research supported by NSFC grant 11901012.

The manuscript of the first version of this paper was done when J. Ye was a Ph.D. student at Texas A\&M University. 
upper planar lattice, growing from an infinite line. SDLA can be seen as a limit of DLA on the upper planar lattice, growing from a long finite line. Mu, Procaccia, and Zhang Mu et al. (2019) consider an edge DLA on the lattice $\mathbb{Z}^{2}$, growing from a long finite line. They show that SDLA is the scaling limit of the bulk of this edge DLA. The result in this paper is crucial to the definition of SDLA in Procaccia et al. (2020) and is a big step towards establishing the convergence of DLA from long line segment to SDLA in Mu et al. (2019).

Several stationary aggregation processes have been studied recently, e.g., stationary Eden model Berger et al. (2014) and stationary internal DLA Antunović and Procaccia (2017). We expect that SDLA has similar geometric properties. For example, one may adopt the approaches in Berger et al. (2014); Antunović and Procaccia (2017), which rely on ergodic theorems and mass transport principle, to show that all trees in SDLA are finite.

1.1. Notations and Definitions. Let $\mathbb{H}=\left\{(x, y) \in \mathbb{Z}^{2}: y \geq 0\right\}$ be the upper half plane including the the x-axis, and $\left\{S_{n}\right\}_{n \geq 0}$ be a 2 -dimensional simple random walk. For any $x \in \mathbb{H}$, we write

$$
x=\left(x^{(1)}, x^{(2)}\right),
$$

where $x^{(i)}$ denotes the i-th coordinate of $x$. For each $n \geq 0$, define the subsets $L_{n} \subset \mathbb{H}$ as follows:

$$
L_{n}=\{(x, n): x \in \mathbb{Z}\},
$$

i.e. $L_{n}$ is the horizontal line of height $n$. For each subset $A \subset \mathbb{H}$, we define the stopping times

$$
\tau_{A}=\min \left\{n \geq 1: S_{n} \in A\right\},
$$

and

$$
\bar{\tau}_{A}=\min \left\{n \geq 0: S_{n} \in A\right\} .
$$

For any $R>0$, let $B(0, R)=\left\{x \in \mathbb{Z}^{2}:\|x\|_{2}<R\right\}$ be the discrete ball of radius $R$, and abbreviate

$$
\tau_{R}=\tau_{B(0, R)}, \bar{\tau}_{R}=\bar{\tau}_{B(0, R)} .
$$

Let $\|\cdot\|_{1}$ be the $\ell_{1}$ norm. We define

$$
\partial^{\text {out }} A:=\left\{y \in \mathbb{H} \backslash A: \exists x \in A,\|x-y\|_{1}=1\right\}
$$

as the outer vertex boundary of $A$, and define

$$
\partial^{\text {in }} A:=\left\{y \in A: \exists x \in \mathbb{H} \backslash A,\|x-y\|_{1}=1\right\}
$$

as the inner vertex boundary of $A$. Let $P_{x}(\cdot)=P\left(\cdot \mid S_{0}=x\right)$. The stationary harmonic measure $\overline{\mathcal{H}}_{A}$ on $\mathbb{H}$ is introduced in Procaccia and Zhang (2019). Let $A \subset \mathbb{H}$ be a connected set. For any edge $e=(x, y)$ of $\mathbb{H}$ with $x \in A$ and $y \in \mathbb{H} \backslash A$, define

$$
\overline{\mathcal{H}}_{A, N}(e)=\sum_{z \in L_{N} \backslash A} P_{z}\left(S_{\bar{\tau}_{A \cup L_{0}}}=x, S_{\bar{\tau}_{A \cup L_{0}}-1}=y\right) .
$$

Since $e=(x, y)$ is an edge in $\mathbb{H}$ with $x \in A$ and $y \in \mathbb{H} \backslash A$, we have $x \in \partial^{\text {in }} A$ and $y \in \partial^{\text {out }} A$. For all $x \in A$, define

$$
\overline{\mathcal{H}}_{A, N}(x)=\sum_{e \text { starting from } x} \overline{\mathcal{H}}_{A, N}(e),
$$

and for all $y \in \mathbb{H} \backslash A$, define

$$
\hat{\mathcal{H}}_{A, N}(y)=\sum_{e \text { starting in } A \text { ending at } y} \overline{\mathcal{H}}_{A, N}(e) .
$$

Proposition 1.1 (Proposition 1 in Procaccia and Zhang, 2019). For any $A$ and e above, there is a finite $\overline{\mathcal{H}}_{A}(e)$ such that

$$
\lim _{N \rightarrow \infty} \overline{\mathcal{H}}_{A, N}(e)=\overline{\mathcal{H}}_{A}(e) .
$$


$\overline{\mathcal{H}}_{A}(e)$ is called the stationary harmonic measure of $e$ with respect to $A$. The limits

$$
\overline{\mathcal{H}}_{A}(x):=\lim _{N \rightarrow \infty} \overline{\mathcal{H}}_{A, N}(x)
$$

and

$$
\hat{\mathcal{H}}_{A}(y):=\lim _{N \rightarrow \infty} \hat{\mathcal{H}}_{A, N}(y)
$$

also exist, and they are called the stationary harmonic measure of $x$ and $y$ with respect to $A$.

Definition 1.2. We say that a set $L_{0} \subset A \subset \mathbb{H}$ has a polynomial sub-linear growth if there exists a constant $\alpha \in(0,1)$ such that

$$
\left|\left\{x=\left(x^{(1)}, x^{(2)}\right) \in A: x^{(2)}>\left|x^{(1)}\right|^{\alpha}\right\}\right|<\infty .
$$

In this paper, we write positive constants as $c, C$, or $c_{0}$, but their values can be different from place to place.

1.2. Main Theorem. Let $\mathcal{H}$ be the regular harmonic measure. The main result of this paper proves the asymptotic equivalence between the stationary harmonic measure of any given point with respect to subset $A$ satisfying Definition 1.2 and the rescaled regular harmonic measure of the same point with respect to the truncations of $A$. To be precise,

Theorem 1.3. For any subset $A$ satisfying Definition 1.2 and any positive integer $n$, let

$$
A_{n}=A \cap([-n, n] \times \mathbb{Z})
$$

be the truncation of $A$ with width $2 n$. There is a constant $C \in(0, \infty)$, independent of the set $A$, such that any point $x \in A \backslash L_{0}$,

$$
C \lim _{n \rightarrow \infty} n \mathcal{H}_{A_{n}}(x)=\overline{\mathcal{H}}_{A}(x) .
$$

Moreover, $C=2 / \lim _{n \rightarrow \infty} n \mathcal{H}_{D_{n}}(0)$, where $D_{n}=([-n, n] \times\{0\}) \cap \mathbb{Z}^{2}$.

Remark 1.4. For points in $L_{0}$, we can replace the regular harmonic measure $\mathcal{H}_{A_{n}}(x)$ in (1.2) by its edge version. I.e., we have for all $x \in L_{0}$,

$$
C \lim _{n \rightarrow \infty} \lim _{\|y\| \rightarrow \infty} n P_{y}\left(S_{\tau_{A_{n}}}=x, S_{\tau_{A_{n}}-1}^{(2)}>0\right)=\overline{\mathcal{H}}_{A}(x) .
$$

Later one can see the proof of (1.3) follows exactly the same argument as the one for (1.2).

The structure of this paper is as follows: We show that stationary harmonic measure is equivalent to a normalized harmonic measure in section 2 (see Theorem 2.8), and the proof of Theorem 1.3 is presented in section 3.

For Theorem 1.3, we first prove its special case when $A=L_{0}$ (so $A_{n}=D_{n}$ in this case). It is not hard to show that $n \mathcal{H}_{D_{n}}(0)$ has finite and positive upper and lower bounds. This and Theorem 2.8 imply the correct scaling for harmonic measure. One of the difficulties is to show that $n \mathcal{H}_{D_{n}}(0)$ converges. We overcome this by showing that $\left|n \mathcal{H}_{D_{n}}(\cdot)-n \mathcal{H}_{D_{n}}(0)\right|$ is small around the neighborhood of 0 , and then pass the limit to its continuous case. Another difficulty is extending the special case to all sets $A$ with polynomial sub-linear growth. Suppose that $L_{0} \subset A \subset \mathbb{H}$ has polynomial sub-linear growth and $x \in \partial^{i n} A \cap A_{n}$. We consider a rectangular box $B$, with a small width and large length, that barely contains $A_{n}$. Suppose that $l$ is a finite line that covers some central part of the top side of $\partial^{\text {in }} B$. A random walk starting from "infinity" in $\mathbb{H}$ (or in $\mathbb{Z}^{2}$ ) must hit $\partial^{\text {in }} B$ before hitting $A$ (or $A_{n}$ ). With small probability the first time a random walk starting from $\partial^{\text {in }} B \backslash l$ in $\mathbb{H}$ (or in $\mathbb{Z}^{2}$ ) hits $A$ (or $A_{n}$ ) is at $x$. Therefore, by the Markov property of random walks, one only need to study the harmonic measure of $l$ with respect to $B$. For any point $y \in l, 2 \mathcal{H}_{B}(y) \approx \mathcal{H}_{D_{n}}(0)$, and the proof of this approximation relies on a discrete version of Beurling estimate in Lawler and Limic (2004) (see Leema 3.17). This explains why $\lim _{n \rightarrow \infty} n \mathcal{H}_{D_{n}}(0)$ appears in the constant $C$ of Theorem 1.3. 


\section{Stationary Harmonic Measure is Equivalent to Normalized Harmonic Measure}

Lemma 2.1. For all $x \in L_{0}, \overline{\mathcal{H}}_{L_{0}}(x)=1$.

Proof: Like Proposition 1 in Procaccia and Zhang (2019), the proof follows a coupling argument by translating one path starting from a fixed point of $L_{N}$ horizontally. For each $N$, let $S_{n}^{(0, N)}$ be a simple random walk in the probability space $P_{(0, N)}(\cdot)$ starting at $(0, N)$, and $S_{n}^{(k, N)}=S_{n}^{(0, N)}+(k, 0)$ for all $k \in \mathbb{Z}$. Note that $S_{n}^{(k, N)}$ is a simple random walk starting at $(k, N)$. Let

$$
\bar{\tau}_{L_{0}}=\inf \left\{n \geq 0: S_{n}^{(0, N)} \in L_{0}\right\}
$$

be a stopping time. Then we have

$$
\bar{\tau}_{L_{0}}=\inf \left\{n \geq 0: S_{n}^{(k, N)} \in L_{0}\right\}
$$

for any $k \in \mathbb{Z}$, and

$$
S_{\bar{\tau}_{L_{0}}}^{(k, N)}=S_{\bar{\tau}_{L_{0}}}^{(0, N)}+(k, 0) .
$$

Hence,

$$
\overline{\mathcal{H}}_{L_{0}, N}(x)=\sum_{k \in \mathbb{Z}} P\left(S_{\bar{\tau}_{L_{0}}}^{(k, N)}=x\right)=1 .
$$

By definition of the stationary harmonic measure,

$$
\overline{\mathcal{H}}_{L_{0}}(x)=\lim _{N \rightarrow \infty} \overline{\mathcal{H}}_{L_{0}, N}(x)=1
$$

We now define a new measure $\widetilde{\mathcal{H}}_{A}(\cdot)$ which is equal to the stationary harmonic measure $\overline{\mathcal{H}}_{A}(\cdot)$ for all sets $A$ with polynomial sub-linear growth. For each $n>0$, we first define

$$
\widetilde{\mathcal{H}}_{A, n}(x)=\pi n P_{(0, n)}\left(S_{\tau_{A \cup L_{0}}}=x\right) .
$$

Lemma 2.2. For all $x=\left(x^{(1)}, 0\right) \in L_{0}$,

$$
\lim _{n \rightarrow \infty} \widetilde{\mathcal{H}}_{L_{0}, n}(x)=1 .
$$

Proof: By Theorem 8.1.2 in Lawler and Limic (2010),

$$
P_{(0, n)}\left(S_{\tau_{L_{0}}}=x\right)=\frac{n}{\pi\left(n^{2}+\left(x^{(1)}\right)^{2}\right)}\left(1+O\left(\frac{n}{n^{2}+\left(x^{(1)}\right)^{2}}\right)\right)+O\left(\frac{1}{\left(n^{2}+\left(x^{(1)}\right)^{2}\right)^{3 / 2}}\right) .
$$

So,

$$
\lim _{n \rightarrow \infty} \widetilde{\mathcal{H}}_{L_{0}, n}(x)=1
$$

Similar to the construction of the stationary harmonic measure $\overline{\mathcal{H}}_{A}(\cdot)$, we want to define a measure $\widetilde{\mathcal{H}}_{A}$ on $\mathbb{H}$ as follows:

$$
\widetilde{\mathcal{H}}_{A}(x):=\lim _{N \rightarrow \infty} \widetilde{\mathcal{H}}_{A, N}(x)
$$

We denote $\widetilde{\mathcal{H}}_{A}(x)$ by in-harmonic measure. We want to show that $\widetilde{\mathcal{H}}_{A}=\overline{\mathcal{H}}_{A}$ if $A$ has polynomial sub-linear growth. Proving that the two measures are equal partly suggests the correct scaling for harmonic measure. Moreover, we provide a good way to approximate stationary harmonic measure. For example, Lemma 2.6 is used in the proof of Lemma 7.1 in Procaccia et al. (2020). We already proved that $\widetilde{\mathcal{H}}_{L_{0}}=\overline{\mathcal{H}}_{L_{0}}$ in Lemma 2.1 and Lemma 2.2. 
Proposition 2.3. Let $A \subset \mathbb{H}$ be a connected finite subset. For any $x \in \mathbb{H}$,

$$
\widetilde{\mathcal{H}}_{A}(x):=\lim _{N \rightarrow \infty} \widetilde{\mathcal{H}}_{A, N}(x)
$$

exists, and $\widetilde{\mathcal{H}}_{A}(x)=\overline{\mathcal{H}}_{A}(x)$.

Proof: Without loss of generality, we assume $x \in \partial^{i n} A$. Let

$$
k=\max \left\{y^{(2)}: y=\left(y^{(1)}, y^{(2)}\right) \in A\right\}
$$

and $n>m>k$ so that $L_{m} \cap A=\emptyset$. By strong Markov property and translation invariance of simple random walk,

$$
\begin{aligned}
& \widetilde{\mathcal{H}}_{A, n}(x) \\
& =\pi n P_{(0, n)}\left(S_{\tau_{A \cup L_{0}}}=x\right) \\
& =\pi n \sum_{y \in L_{m}} P_{(0, n)}\left(S_{\tau_{L_{m}}}=y\right) P_{y}\left(S_{\tau_{A \cup L_{0}}}=x\right) \\
& =\frac{n}{n-m} \sum_{y \in L_{m}} P_{y}\left(S_{\tau_{A \cup L_{0}}}=x\right)\left[\pi(n-m) P_{(0, n)}\left(S_{\tau_{L_{m}}}=y\right)\right] \\
& =\frac{n}{n-m} \sum_{y \in L_{m}} P_{y}\left(S_{\tau_{A \cup L_{0}}}=x\right) \widetilde{\mathcal{H}}_{L_{0}, n-m}\left(y_{0}\right),
\end{aligned}
$$

where $y_{0}=\left(y^{(1)}, 0\right)$. Then by Dominated Convergence Theorem and Lemma 2.2,

$$
\begin{aligned}
& \lim _{n \rightarrow \infty} \widetilde{\mathcal{H}}_{A, n}(x) \\
& =\lim _{n \rightarrow \infty} \sum_{y \in L_{m}} P_{y}\left(S_{\tau_{A \cup L_{0}}}=x\right) \frac{n}{n-m} \widetilde{\mathcal{H}}_{L_{0}, n-m}\left(y_{0}\right) \\
& =\sum_{y \in L_{m}} P_{y}\left(S_{\tau_{A \cup L_{0}}}=x\right)\left[\lim _{n \rightarrow \infty} \frac{n}{n-m} \widetilde{\mathcal{H}}_{L_{0}, n-m}\left(y_{0}\right)\right] \\
& =\sum_{y \in L_{m}} P_{y}\left(S_{\tau_{A \cup L_{0}}}=x\right) \\
& =\overline{\mathcal{H}}_{A, m}(x) .
\end{aligned}
$$

We can apply Dominated Convergence Theorem in equation (2.2) because $\widetilde{\mathcal{H}}_{L_{0}, n-m}\left(y_{0}\right)$ is uniformly bounded from above for all $n$ and $y_{0} \in \mathbb{Z}$ by Theorem 8.1.2 of Lawler and Limic (2010) and the fact that $\widetilde{\mathcal{H}}_{L_{0}, n-m}(0) \geq \widetilde{\mathcal{H}}_{L_{0}, n-m}\left(y_{0}\right)$ for all $y_{0} \in L_{0}$. We claim that $\overline{\mathcal{H}}_{A, m}(x)=\overline{\mathcal{H}}_{A}(x)$. Let $m_{1}>m$. 
By strong Markov property and Lemma 2.1,

$$
\begin{aligned}
& \overline{\mathcal{H}}_{A, m_{1}}(x) \\
& =\sum_{y \in L_{m_{1}}} P_{y}\left(S_{\tau_{A \cup L_{0}}}=x\right) \\
& =\sum_{y \in L_{m_{1}}} \sum_{z \in L_{m}} P_{y}\left(S_{\tau_{L_{m}}}=z\right) P_{z}\left(S_{\tau_{A \cup L_{0}}}=x\right) \\
& =\sum_{z \in L_{m}} P_{z}\left(S_{\tau_{A \cup L_{0}}}=x\right)\left[\sum_{y \in L_{m_{1}}} P_{y}\left(S_{\tau_{L_{m}}}=z\right)\right] \\
& =\sum_{z \in L_{m}} P_{z}\left(S_{\tau_{A \cup L_{0}}}=x\right) \overline{\mathcal{H}}_{L_{0}, m_{1}-m}\left(z^{\prime}\right) \\
& =\sum_{z \in L_{m}} P_{z}\left(S_{\tau_{A \cup L_{0}}}=x\right) \\
& =\overline{\mathcal{H}}_{A, m}(x)
\end{aligned}
$$

where $z^{\prime}=z-(0, m)$. Hence,

$$
\widetilde{\mathcal{H}}_{A}(x)=\overline{\mathcal{H}}_{A, m}(x)=\lim _{N \rightarrow \infty} \overline{\mathcal{H}}_{A, N}(x)=\overline{\mathcal{H}}_{A}(x) .
$$

For any positive integer $n$, consider the following rectangle in $\mathbb{Z}^{2}$ :

$$
I_{n}=[-n, n] \times[0, n]
$$

with height $n$ and width $2 n$. It is easy to see that $I_{n} \subset B(0,2 n)$. Moreover, we let $\partial^{i n} I_{n}$ be the inner vertex boundary of $A_{n}$, and let

$$
\partial_{l}^{i n} I_{n}=\{-n\} \times[1, n], \quad \partial_{r}^{i n} I_{n}=\{n\} \times[1, n], \quad \partial_{u}^{i n} I_{n}=[-n, n] \times\{n\}, \quad \partial_{b}^{i n} I_{n}=[-n, n] \times\{0\}
$$

be the four edges of $\partial^{i n} I_{n}$.

Let $\left\{S_{n}\right\}_{n \geq 0}$ be a simple random walk starting from 0 and denote by $P_{0}$ the probability distribution of $S_{n}$. Define the stopping time

$$
T_{n}=\inf \left\{k>0, S_{k} \in \partial^{i n} I_{n}\right\} .
$$

Using simple combinatorial arguments, we prove the following lemma:

Lemma 2.4. For any integer $n>1$

$$
P_{0}\left(S_{T_{n}} \in \partial_{u}^{i n} I_{n}\right) \geq P_{0}\left(S_{T_{n}} \in \partial_{l}^{i n} I_{n} \cup \partial_{r}^{i n} I_{n}\right)
$$

Proof: Let $\partial_{u,+}^{i n} I_{n}=[1, n] \times\{n\}$ and $\partial_{u,-}^{i n} I_{n}=[-n,-1] \times\{n\}$ be the left and right half of $\partial_{u}^{i n} I_{n}$. By symmetry it suffices to prove that

$$
P_{0}\left(S_{T_{n}} \in \partial_{u,+}^{i n} I_{n}\right) \geq P_{0}\left(S_{T_{n}} \in \partial_{r}^{i n} I_{n}\right) .
$$

By definition, we have

$$
P_{0}\left(S_{T_{n}} \in \partial_{u,+}^{i n} I_{n}\right)=\sum_{k=1}^{\infty} P_{0}\left(S_{k} \in \partial_{u,+}^{i n} I_{n}, T_{n}=k\right)
$$

and

$$
P_{0}\left(S_{T_{n}} \in \partial_{r}^{i n} I_{n}\right)=\sum_{k=1}^{\infty} P_{0}\left(S_{k} \in \partial_{r}^{i n} I_{n}, T_{n}=k\right)
$$


Moreover, for each $k$,

where

$$
P_{0}\left(S_{k} \in \partial_{u,+}^{i n} I_{n}, T_{n}=k\right)=\frac{\left|\mathcal{U}_{n, k}^{+}\right|}{4^{k}}, \quad P_{0}\left(S_{k} \in \partial_{r}^{i n} I_{n}, T_{n}=k\right)=\frac{\left|\mathcal{R}_{n, k}\right|}{4^{k}},
$$

$$
\begin{array}{r}
\mathcal{U}_{n, k}^{+}=\left\{\left(a_{0}, a_{1}, \cdots, a_{k}\right), \text { such that } a_{0}=0,\left\|a_{i+1}-a_{i}\right\|=1, \forall i=0,1, \cdots, k-1,\right. \\
\left.a_{j} \in A_{n} \backslash \partial^{i n} A_{n}, \forall j=1,2, \cdots, k-1, a_{k} \in \partial_{u,+}^{i n} I_{n}\right\}
\end{array}
$$

and

$$
\begin{array}{r}
\mathcal{R}_{n, k}=\left\{\left(a_{0}, a_{1}, \cdots, a_{k}\right), \text { such that } a_{0}=0,\left\|a_{i+1}-a_{i}\right\|=1, \forall i=0,1, \cdots, k-1,\right. \\
\left.\qquad a_{j} \in A_{n} \backslash \partial^{i n} A_{n}, \forall j=1,2, \cdots, k-1, a_{k} \in \partial_{r}^{i n} I_{n}\right\}
\end{array}
$$

give the subsets of the random walk trajectories in events $\left\{S_{T_{n}} \in \partial_{u,+}^{i n} I_{n}\right\}$ and $\left\{S_{T_{n}} \in \partial_{r}^{i n} I_{n}\right\}$.

Thus in order to show (2.5), we construct a one-to-one mapping $\varphi$ between the trajectories in $\mathcal{R}_{n, k}$ and $\mathcal{U}_{n, k}^{+}$. For any trajectory $\vec{a}=\left(a_{0}, a_{1}, \cdots, a_{k}\right) \in \mathcal{R}_{n, k}$, define

$$
m(\vec{a})=\sup \left\{i \geq 0, a_{i}^{(1)}=a_{i}^{(2)}\right\}
$$

to be the last point in the trajectory lying on the diagonal. Here $a_{i}^{(1)}$ and $a_{i}^{(2)}$ are the two coordinates of $a_{i}$. In this paper, we use the convention that $\sup \{\emptyset\}=-\infty$. Then it is easy to see that $0 \in\left\{i \geq 0, a_{i}^{(1)}=a_{i}^{(2)}\right\}$ and thus $m(\vec{a}) \geq 0$ and $m(\vec{a})<k$. The reason of the latter inequality is that suppose $m(\vec{a})=k$, then we must have $a_{k}=(n, n)$ which implies that $a_{k-1}=(n-1, n)$ or $(n, n-1)$, which contradicts with the definition of $\vec{a}$.

Now we can define

$$
\varphi(\vec{a})=\vec{a}^{\prime}=\left(a_{0}^{\prime}, a_{1}^{\prime}, \cdots, a_{k}^{\prime}\right)
$$

such that

- $a_{i}^{\prime}=a_{i}$ for all $i \leq m(\vec{a})$.

- $a_{i}^{\prime}=\left(a_{i}^{(2)}, a_{i}^{(1)}\right)$ for all $i>m(\vec{a})$.

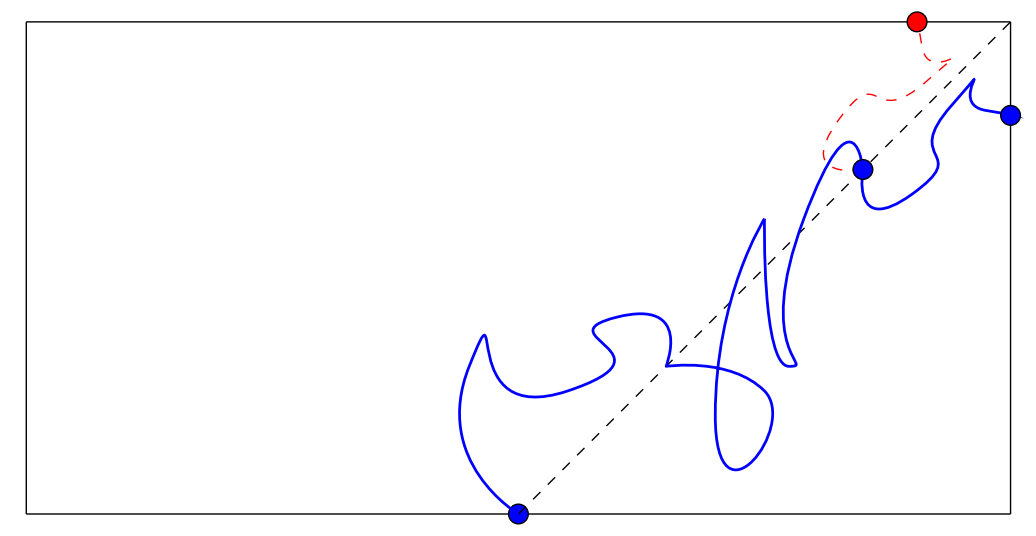

FIGURE 2.1. mapping between trajectories in $\mathcal{R}_{n, k}$ and $\mathcal{U}_{n, k}^{+}$

I.e., we reflect the trajectory after the last time it visits the diagonal line $x=y$. By definition

$$
\left(a_{m(\vec{a})+1}, a_{m(\vec{a})+2}, \cdots, a_{k-1}\right)
$$

stays within $\left\{(x, y) \in \mathbb{Z}^{2}, 0<y<x<n\right\}$, while $a_{k} \in R_{n}$. Thus, under reflection we have

$$
\left(a_{m(\vec{a})+1}^{\prime}, a_{m(\vec{a})+2}^{\prime}, \cdots, a_{k-1}^{\prime}\right)
$$


stays within $\left\{(x, y) \in \mathbb{Z}^{2}, 0<x<y<n\right\}$, while $a_{k}^{\prime} \in U_{n, k}^{+}$, which implies that $\vec{a}^{\prime} \in \mathcal{U}_{n, k}^{+}$.

On the other hand, suppose we have two trajectories $\vec{a}$ and $\vec{b}$ both in $\mathcal{R}_{n, k}$ such that $\varphi(\vec{a})=\varphi(\vec{b})$. Then one must have $m(\vec{a})=m(\vec{b})=m$ and $a_{i}=b_{i}$ for all $i \leq m$. Moreover, for all $i>m$, we have

$$
\left(a_{i}^{(2)}, a_{i}^{(1)}\right)=a_{i}^{\prime}=b_{i}^{\prime}=\left(b_{i}^{(2)}, b_{i}^{(1)}\right)
$$

which also implies that $a_{i}=b_{i}$. Thus, we have shown that $\varphi(\vec{a})=\varphi(\vec{b})$ if and only if $\vec{a}=\vec{b}$ and $\varphi$ is a one-to-one mapping, which conclude the proof of this lemma.

We define

$$
F_{m}=F_{m, \alpha}=\left\{-\left\lfloor m^{1 / \alpha}\right\rfloor,\left\lfloor m^{1 / \alpha}\right\rfloor\right\} \times \mathbb{Z}_{\geq 0}
$$

as two vertical lines on $\mathbb{H}$.

Lemma 2.5. Fix $x \in \mathbb{H}$, then for all sufficiently large $m$,

$$
P_{x}\left(\tau_{F_{m, \alpha}}<\tau_{L_{0}}\right) \leq c m^{-1 / \alpha} .
$$

Proof: Let $m>4\left|x_{1}\right|$, and $x^{\prime}=\left(x^{(1)}, 0\right)$. There exists a constant $C>0$ independent of $m$ such that

$$
C P_{x}\left(\tau_{F_{m, \alpha}}<\tau_{L_{0}}\right) \leq P_{x^{\prime}}\left(\tau_{F_{m, \alpha}}<\tau_{L_{0}}\right) .
$$

By translation invariance of simple random walk, we have

$$
P_{x^{\prime}}\left(\tau_{F_{m, \alpha}}<\tau_{L_{0}}\right) \leq P_{0}\left(\tau_{I_{\left\lfloor m^{1 / \alpha} / 2\right\rfloor}}<\tau_{L_{0}}\right) .
$$

By Lemma 2.4,

$$
P_{0}\left(\tau_{I_{\left\lfloor m^{1 / \alpha} / 2\right\rfloor}}<\tau_{L_{0}}\right) \leq 2 P_{0}\left(\tau_{L_{\left\lfloor m^{1 / \alpha} / 2\right\rfloor}}<\tau_{L_{0}}\right) \leq \mathrm{cm}^{-1 / \alpha}
$$

The next lemma claims that $\overline{\mathcal{H}}_{A}$ is concentrated on the part arising from random walks starting from $y \in L_{m}$ such that $\left|y^{(1)}\right| \leq\left\lfloor m^{1 / \alpha}\right\rfloor$.

Lemma 2.6. Let $A \subset \mathbb{H}$ be an infinite set that has polynomial sub-linear growth with parameter $\alpha \in(0,1)$. Let $1>\alpha_{1}=(\alpha+1) / 2>\alpha$, then for any $x \in \mathbb{H}$,

$$
\lim _{m \rightarrow \infty}\left|\sum_{y \in L_{m},\left|y^{(1)}\right| \leq\left\lfloor m^{1 / \alpha_{1}}\right\rfloor} P_{y}\left(S_{\tau_{A} \cup L_{0}}=x\right)-\overline{\mathcal{H}}_{A, m}(x)\right|=0 .
$$

Proof: Note that $\left\{y \in L_{n},\left|y^{(1)}\right| \leq\left\lfloor n^{1 / \alpha_{1}}\right\rfloor\right\} \cap A=\emptyset$. Following the argument in Kesten (1987, Lemma 2) on time reversibility and symmetry of simple random walk, we have

$$
\begin{aligned}
& P_{y}\left(\tau_{x}=k, S_{1}, \cdots, S_{k-1} \notin\{x\} \cup L_{0}\right) \\
& =P_{x}\left(\tau_{y}=k, S_{1}, \cdots, S_{k-1} \notin\{x\} \cup L_{0}\right) \\
& =P_{x}\left(S_{k}=y, \tau_{\{x\} \cup L_{0}}>k\right) .
\end{aligned}
$$


Taking the summation over all $k$, we have

$$
\begin{aligned}
& P_{y}\left(\tau_{x} \leq \tau_{L_{0}}\right) \\
& =\sum_{k=1}^{\infty} P_{y}\left(\tau_{x}=k, S_{1}, \cdots, S_{k-1} \notin\{x\} \cup L_{0}\right) \\
& =\sum_{k=1}^{\infty} P_{x}\left(S_{k}=y, \tau_{\{x\} \cup L_{0}}>k\right) \\
& \leq E_{x}\left[\text { number of visits to } y \text { in the time interval }\left[0, \tau_{\{x\} \cup L_{0}}\right)\right] \\
& \leq E_{x}\left[\text { number of visits to } y \text { in the time interval }\left[0, \tau_{L_{0}}\right)\right] .
\end{aligned}
$$

Therefore,

$$
\begin{aligned}
& \lim _{m \rightarrow \infty} \sum_{y \in L_{m} \backslash A,\left|y^{(1)}\right| \geq\left\lceil m^{\left.1 / \alpha_{1}\right\rceil}\right.} P_{y}\left(S_{\tau_{A}}=x\right) \\
& \leq \lim _{m \rightarrow \infty} \sum_{y \in L_{m} \backslash A,\left|y^{(1)}\right| \geq\left\lceil m^{\left.1 / \alpha_{1}\right\rceil}\right.} P_{y}\left(\tau_{x} \leq \tau_{L_{0}}\right) \\
& \leq \lim _{m \rightarrow \infty} \sum_{y \in L_{m} \backslash A,\left|y^{(1)}\right| \geq\left\lceil m^{1 / \alpha_{1}}\right\rceil} E_{x}\left[\text { number of visits to } y \text { in the time interval }\left[0, \tau_{L_{0}}\right)\right] \\
& \leq \lim _{m \rightarrow \infty} E_{x}\left[\text { number of visits to } G_{m, \alpha_{1}} \text { in the time interval }\left[0, \tau_{L_{0}}\right)\right],
\end{aligned}
$$

where $G_{m, \alpha_{1}}=\left\{y \in L_{m}:\left|y^{(1)}\right| \geq\left\lceil m^{1 / \alpha_{1}}\right\rceil\right\}$. By Lemma 2.5, we have

$$
\begin{aligned}
& \lim _{m \rightarrow \infty}\left|\sum_{y \in L_{m} \backslash A,\left|y^{(1)}\right| \geq\left\lceil m^{\left.1 / \alpha_{1}\right\rceil}\right.} P_{y}\left(S_{\tau_{A}}=x\right)\right| \\
& \leq \lim _{m \rightarrow \infty} E_{x}\left[\text { number of visits to } G_{m, \alpha_{1}} \text { in the time interval }\left[0, \tau_{L_{0}}\right)\right] \\
& \leq \lim _{m \rightarrow \infty} 4 m P_{x}\left(\tau_{G_{m, \alpha_{1}}}<\tau_{L_{0}}\right) \\
& \leq \lim _{m \rightarrow \infty} 4 m P_{x}\left(\tau_{F_{m, \alpha_{1}}}<\tau_{L_{0}}\right) \\
& =0 .
\end{aligned}
$$

The proof is complete.

Lemma 2.7. Let $A \subset \mathbb{H}$ be an infinite set that has polynomial sub-linear growth with parameter $\alpha \in(0,1)$. Let $1>\alpha_{1}=(\alpha+1) / 2>\alpha$, then for all $x \in \mathbb{H}$ and for all $\epsilon>0$ and for $m$ and $n=n(m)$ large enough, we have

$$
\left|\sum_{y \in L_{m},\left|y^{(1)}\right| \leq\left\lfloor m^{1 / \alpha_{1}}\right\rfloor} P_{y}\left(S_{\tau_{A} \cup L_{0}}=x\right)-\widetilde{\mathcal{H}}_{A, n}(x)\right|<\epsilon .
$$

Proof: Fix $x \in \mathbb{H}$ and $\epsilon>0$. Let $l=\max \left\{y^{(2)}: y \in A, y^{(2)}>\left|y^{(1)}\right|^{\alpha}\right\}$. Assume that $n$ and $m$ are large with $n>m>\max \left\{l, x^{(2)}\right\}$. Let $\alpha_{1}=(\alpha+1) / 2$ as defined in Lemma 2.6. By strong Markov 
property, we have

$$
\begin{aligned}
& \widetilde{\mathcal{H}}_{A, n}(x) \\
& =\pi n P_{(0, n)}\left(S_{\tau_{A}}=x\right) \\
& =\sum_{y \in L_{m} \backslash A} \pi n P_{(0, n)}\left(S_{\tau_{A \cup L_{m}}}=y\right) P_{y}\left(S_{\tau_{A}}=x\right) \\
& \leq \sum_{y \in L_{m},\left|y^{(1)}\right| \leq\left\lfloor m^{1 / \alpha_{1}}\right\rfloor} \pi n P_{(0, n)}\left(S_{\tau_{A \cup L_{m}}}=y\right) P_{y}\left(S_{\tau_{A}}=x\right)+c \sum_{y \in L_{m} \backslash A,\left|y^{(1)}\right| \geq\left\lceil m^{1 / \alpha_{1}}\right\rceil} P_{y}\left(S_{\tau_{A}}=x\right),
\end{aligned}
$$

where $c>0$ is a constant. The last inequality of equation (2.10) is using Theorem 8.1.2 in Lawler and Limic (2010) and the fact that

$$
P_{(0, n)}\left(S_{\tau_{A \cup L m}}=y\right) \leq P_{(0, n)}\left(S_{\tau_{L_{m}}}=y\right) .
$$

By Lemma 2.6, we know

$$
\lim _{m \rightarrow \infty} \sum_{y \in L_{m} \backslash A,\left|y^{(1)}\right| \geq\left\lceil m^{1 / \alpha_{1}}\right\rceil} P_{y}\left(S_{\tau_{A}}=x\right)=0 .
$$

Thus, there exists a $M_{1}>\max \left\{l, x^{(2)}\right\}$ such that for all $m>M_{1}$ and all sufficiently large $n>m$,

$$
\left|\widetilde{\mathcal{H}}_{A, n}(x)-\sum_{y \in L_{m},\left|y^{(1)}\right| \leq\left\lfloor m^{1 / \alpha_{1}}\right\rfloor} \pi n P_{(0, n)}\left(S_{\tau_{A \cup L_{m}}}=y\right) P_{y}\left(S_{\tau_{A}}=x\right)\right|<\frac{\epsilon}{2} .
$$

Denote the set

$$
\widetilde{A}_{m}=\left\{x \in \mathbb{H}: x^{(1)}>\left\lfloor m^{1 / \alpha}\right\rfloor, m \leq x^{(2)} \leq\left|x^{(1)}\right|^{\alpha}\right\} .
$$

Note that $\widetilde{A}_{m}$ contains the part of $A$ that is above the horizontal line $L_{m}$. For $y \in L_{m}$ such that $\left|y^{(1)}\right| \leq m^{1 / \alpha_{1}}$, we have

$$
P_{(0, n)}\left(S_{\tau_{A \cup L m}}=y\right) \leq P_{(0, n)}\left(S_{\tau_{L_{m}}}=y\right),
$$

while

$$
\begin{aligned}
P_{(0, n)}\left(S_{\tau_{A \cup L_{m}}}=y\right) & \geq P_{(0, n)}\left(S_{\tau_{\widetilde{A}_{m} \cup L_{m}}}=y\right) \\
& =P_{(0, n)}\left(S_{\tau_{L_{m}}}=y\right)-\sum_{z \in \widetilde{A}_{m}} P_{(0, n)}\left(S_{\tau_{\widetilde{A}_{m} \cup L_{m}}}=z\right) P_{z}\left(S_{\tau_{L_{m}}}=y\right) .
\end{aligned}
$$

Note that for $z \in \widetilde{A}_{m}, P_{(0, n)}\left(S_{\tau_{\widetilde{A}_{m} \cup L_{m}}}=z\right)=0$ unless $z$ is in the upper inner boundary of $\widetilde{A}_{m}$, i.e., $z=\left(k,\left\lfloor k^{\alpha}\right\rfloor\right) \in \partial^{\text {in }} \widetilde{A}_{m}$ for some $k>\left\lfloor m^{1 / \alpha}\right\rfloor$. Suppose $z=\left(k,\left\lfloor k^{\alpha}\right\rfloor\right) \in \partial^{\text {in }} \widetilde{A}_{m}$ with $k>\left\lfloor m^{1 / \alpha}\right\rfloor$. Let $y \in L_{m}$ such that $\left|y^{(1)}\right| \leq m^{1 / \alpha_{1}}$. By Theorem 8.1.2 in Lawler and Limic Lawler and Limic (2010), we have

$$
\begin{aligned}
& P_{z}\left(S_{\tau_{L_{m}}}=y\right) \\
& \leq \frac{c\left(\left\lfloor k^{\alpha}\right\rfloor-m\right)}{\left(\left\lfloor k^{\alpha}\right\rfloor-m\right)^{2}+\left(k-\left\lfloor m^{1 / \alpha_{1}}\right\rfloor\right)^{2}} \\
& \leq \frac{c\left(k^{\alpha}-m\right)}{\left(\left\lfloor k^{\alpha}\right\rfloor-m\right)^{2}+\left(k-m^{1 / \alpha_{1}}\right)^{2}} .
\end{aligned}
$$


Thus,

$$
\begin{aligned}
& \sum_{z \in \widetilde{A}_{m}} P_{(0, n)}\left(S_{\tau_{\widetilde{A}_{m} \cup L_{m}}}=z\right) P_{z}\left(S_{\tau_{L_{m}}}=y\right) \\
& \leq \sum_{z \in \widetilde{A}_{m}} P_{z}\left(S_{\tau_{L_{m}}}=y\right) \\
& \leq c \sum_{k=\left\lceil m^{1 / \alpha}\right\rceil}^{\infty} \frac{k^{\alpha}-m}{\left(\left\lfloor k^{\alpha}\right\rfloor-m\right)^{2}+\left(k-m^{1 / \alpha_{1}}\right)^{2}} \\
& \leq c \sum_{s=1}^{\infty} \frac{\left(s+m^{1 / \alpha}+1\right)^{\alpha}-m}{\left(\left\lfloor\left(s+\left\lfloor m^{1 / \alpha}\right\rfloor\right)^{\alpha}\right\rfloor-m\right)^{2}+\left(s+m^{1 / \alpha}-m^{1 / \alpha_{1}}\right)^{2}}
\end{aligned}
$$

It's easy to see that the sum above converges and goes to 0 if $m$ goes to infinity. Consider the sum

$$
S:=c m^{3 /(2 \alpha)-1 / 2} \sum_{s=1}^{\infty} \frac{\left(s+m^{1 / \alpha}+1\right)^{\alpha}-m}{\left(\left\lfloor\left(s+\left\lfloor m^{1 / \alpha}\right\rfloor\right)^{\alpha}\right\rfloor-m\right)^{2}+\left(s+m^{1 / \alpha}-m^{1 / \alpha_{1}}\right)^{2}} .
$$

Note that

$$
\begin{aligned}
& c m^{3 /(2 \alpha)-1 / 2} \sum_{s=1}^{\infty} \frac{\left(s+m^{1 / \alpha}+1\right)^{\alpha}-m}{\left(\left\lfloor\left(s+\left\lfloor m^{1 / \alpha}\right\rfloor\right)^{\alpha}\right\rfloor-m\right)^{2}+\left(s+m^{1 / \alpha}-m^{1 / \alpha_{1}}\right)^{2}} \\
& \leq c m^{3 /(2 \alpha)-1 / 2} \sum_{s=1}^{\infty} \frac{\left(s+m^{1 / \alpha}+1\right)^{\alpha}-m}{\left(s+m^{1 / \alpha}-m^{1 / \alpha_{1}}\right)^{2}} .
\end{aligned}
$$

For all $0<\alpha<1$, there is a $M>0$ large enough such that for all $s>0$ and $m^{\prime}>M$,

$$
\left.\frac{\partial}{\partial m}\left(c m^{3 /(2 \alpha)-1 / 2} \sum_{s=1}^{\infty} \frac{\left(s+m^{1 / \alpha}+1\right)^{\alpha}-m}{\left(s+m^{1 / \alpha}-m^{1 / \alpha_{1}}\right)^{2}}\right)\right|_{m=m^{\prime}}<0
$$

So the sum $S$ goes to 0 if $m$ goes to infinity. Hence, we can take $n=\left\lfloor m^{3 /(2 \alpha)-1 / 2}\right\rfloor$. Note that $3 /(2 \alpha)-1 / 2>1 / \alpha$. For any $y \in L_{m}$ with $\left|y^{(1)}\right| \leq\left\lfloor m^{1 / \alpha_{1}}\right\rfloor$, we have

$$
\lim _{m \rightarrow \infty} n \sum_{z \in \widetilde{A}_{m}} P_{(0, n)}\left(S_{\tau_{\widetilde{A}_{m} \cup L_{m}}}=z\right) P_{z}\left(S_{\tau_{L_{m}}}=y\right)=0
$$

and

$$
\lim _{m \rightarrow \infty} \pi n P_{(0, n)}\left(S_{\tau_{A \cup L_{m}}}=y\right)=1 .
$$

Now fix $N>\max \left\{l, x_{2}\right\}$. From the proof of Theorem 1 in Procaccia and Zhang (2019), we know that the sequence $H_{A, j}(x)$ is decreasing for $j \geq N$. There exists a $M_{2}>N$ such that for all $m>M_{2}$,

$$
\left|\pi n P_{(0, n)}\left(S_{\tau_{A \cup L_{m}}}=y\right)-1\right|<\frac{\epsilon}{2 H_{A, N}(x)} .
$$

Therefore,

$$
\left|\sum_{y \in L_{m},\left|y^{(1)}\right| \leq\left\lfloor m^{1 / \alpha}\right\rfloor}\left(\pi n P_{(0, n)}\left(S_{\tau_{A \cup L m}}=y\right)-1\right) P_{y}\left(S_{\tau_{A}}=x\right)\right|<\frac{\epsilon}{2} .
$$

Now take $m>\max \left\{M_{1}, M_{2}\right\}$, and the proof is complete.

The following theorem is a direct consequence of Lemma 2.6 and Lemma 2.7. 
Theorem 2.8. Let $A \subset \mathbb{H}$ be an infinite set that has polynomial sub-linear growth. For any $x \in \mathbb{H}$,

$$
\widetilde{\mathcal{H}}_{A}(x):=\lim _{N \rightarrow \infty} \widetilde{\mathcal{H}}_{A, N}(x)
$$

exists, and $\widetilde{\mathcal{H}}_{A}(x)=\overline{\mathcal{H}}_{A}(x)$.

Proof: Let $\epsilon>0$. By Lemma 2.6 and Lemma 2.7, there is an $M>0$ such that for all $m>M$,

$$
\left|\overline{\mathcal{H}}_{A, m}(x)-\widetilde{\mathcal{H}}_{A, m}(x)\right|<\epsilon .
$$

We know

Hence,

$$
\lim _{m \rightarrow \infty} \overline{\mathcal{H}}_{A, m}(x)=\overline{\mathcal{H}}_{A}(x) .
$$

exists and $\widetilde{\mathcal{H}}_{A}(x)=\overline{\mathcal{H}}_{A}(x)$.

$$
\widetilde{\mathcal{H}}_{A}(x):=\lim _{m \rightarrow \infty} \widetilde{\mathcal{H}}_{A, m}(x)
$$

\section{Proof of the Main Theorem}

In order to prove Theorem 1.3, we first show its special case when $A=L_{0}$, which can be stated as the following result on the asymptotic of regular harmonic measures: Let $D_{n}=[-n, n] \times\{0\}$ to be the horizontal line segment of interest. In this section we proved that

Theorem 3.1. There is a constant $c \in(0, \infty)$ such that

$$
\lim _{n \rightarrow \infty} n \mathcal{H}_{D_{n}}(0)=c .
$$

The structure of this section is as follows: In subsections 3.1 and 3.2 we outline the proof of Theorem 3.1 and Theorem 1.3. Then in the following subsections, we give the detailed proof of the required propositions and lemmas.

3.1. Proof of Theorem 3.1. Theorem 3.1 can be proved according to the following outline: First, we show that $n \mathcal{H}_{D_{n}}(0)$ has finite and positive upper and lower limits:

Proposition 3.2. There is a constant $C \in(0, \infty)$ such that

$$
\limsup _{n \rightarrow \infty} n \mathcal{H}_{D_{n}}(0) \leq C \text {. }
$$

Proposition 3.3. There is a constant $c \in(0, \infty)$ such that

$$
\liminf _{n \rightarrow \infty} n \mathcal{H}_{D_{n}}(0) \geq c .
$$

The two propositions above guarantee that the decaying rate of $\mathcal{H}_{D_{n}}(0)$ is of order $1 / n$. To show limsup = lim inf, we further show the following coupling result:

Proposition 3.4. For any $\epsilon>0$, there is a $\delta>0$ such that for all sufficiently large $n$ and any $x \in[-\delta n, \delta n] \times\{0\}$, we have

$$
\left|\mathcal{H}_{D_{n}}(0)-\mathcal{H}_{D_{n}}(x)\right|<\frac{\epsilon}{n}
$$

Let $\bar{B}(0, R)=\left\{x \in \mathbb{R}^{2}:\|x\|_{2}<R\right\}$ be the continuous ball of radius $R$ in $\mathbb{R}^{2}$. For standard Brownian motion $B(t)$ and subset $A \subset \mathbb{R}^{2}$, define the stopping time

$$
T_{A}=\inf \{t \geq 0: B(t) \in A\} .
$$

For subset $A \subset \mathbb{R}^{2}, \mathrm{H}_{A}$ denotes the continuous harmonic measure with respect to $A$.

Lemma 3.5. Fix $\delta \in(0,1)$, then

$$
\lim _{n \rightarrow \infty} \mathcal{H}_{D_{n}}([-\delta n, \delta n] \times\{0\})=\mathrm{H}_{[-1,1] \times\{0\}}([-\delta, \delta] \times\{0\}) .
$$


Once one has shown Propositions 3.2-3.5, the proof of Theorem 3.1 is mostly straightforward. Now suppose the limit in (3.1) does not exist. Then by Proposition 3.2 we must have

$$
0<\liminf _{n \rightarrow \infty} n \mathcal{H}_{D_{n}}(0)<\limsup _{n \rightarrow \infty} n \mathcal{H}_{D_{n}}(0)<\infty .
$$

Let

$$
\epsilon_{0}=\frac{\limsup _{n \rightarrow \infty} n \mathcal{H}_{D_{n}}(0)-\liminf _{n \rightarrow \infty} n \mathcal{H}_{D_{n}}(0)}{5}>0 .
$$

By Proposition 3.4, there are $\delta_{0}>0$ and $N_{0}<\infty$ such that for all $n>N_{0}$ and any $x \in\left[-\delta_{0} n, \delta_{0} n\right] \times$ $\{0\}$,

$$
\left|\mathcal{H}_{D_{n}}(0)-\mathcal{H}_{D_{n}}(x)\right|<\frac{\epsilon}{n}
$$

Moreover, for any $N>N_{0}$, there are $n_{1}, n_{2}>N$ such that

$$
n_{1} \mathcal{H}_{D_{n_{1}}}(0)<\liminf _{n \rightarrow \infty} n \mathcal{H}_{D_{n}}(0)+\epsilon_{0},
$$

and that

$$
n_{2} \mathcal{H}_{D_{n_{2}}}(0)>\limsup _{n \rightarrow \infty} n \mathcal{H}_{D_{n}}(0)-\epsilon_{0}
$$

At the same time, for the $\delta_{0}>0$ defined above,

$$
\begin{aligned}
\mathcal{H}_{D_{n_{1}}}\left(\left[-\delta_{0} n_{1}, \delta_{0} n_{1}\right] \times\{0\}\right) & =\sum_{x \in\left[-\delta_{0} n_{1}, \delta_{0} n_{1}\right] \times\{0\}} \mathcal{H}_{D_{n_{1}}}(x) \\
& \leq \frac{\left\lfloor\delta_{0} n_{1}\right\rfloor+1}{n_{1}}\left[\liminf _{n \rightarrow \infty} n \mathcal{H}_{D_{n}}(0)+2 \epsilon_{0}\right]
\end{aligned}
$$

and

But by Lemma 3.5,

$$
\begin{aligned}
\mathcal{H}_{D_{n_{2}}}\left(\left[-\delta_{0} n_{2}, \delta_{0} n_{2}\right] \times\{0\}\right) & =\sum_{x \in\left[-\delta_{0} n_{2}, \delta_{0} n_{2}\right] \times\{0\}} \mathcal{H}_{D_{n_{2}}}(x) \\
& \geq \frac{\left\lfloor\delta_{0} n_{2}\right\rfloor+1}{n_{2}}\left[\limsup _{n \rightarrow \infty} n \mathcal{H}_{D_{n}}(0)-2 \epsilon_{0}\right] .
\end{aligned}
$$

$$
\lim _{n \rightarrow \infty} \mathcal{H}_{D_{n}}\left(\left[-\delta_{0} n, \delta_{0} n\right] \times\{0\}\right)=\mathrm{H}_{[-1,1] \times\{0\}}\left(\left[-\delta_{0}, \delta_{0}\right] \times\{0\}\right),
$$

which contradicts (3.6) and (3.7).

3.2. Proof of Theorem 1.3. Define $\alpha_{1}=(1+\alpha) / 2 \in(0,1)$ and $B o x(n)=[-n, n] \times\left[0,\left\lfloor n^{\alpha_{1}}\right\rfloor\right]$. Recall the definition of regular harmonic measure and the fact that $A_{n} \subset \operatorname{Box}(n)$ for all sufficiently large $n$. For any $x \in A \backslash L_{0}$,

$$
\mathcal{H}_{A_{n}}(x)=\sum_{y \in \partial^{i n} \operatorname{Box}(n)} \mathcal{H}_{B o x(n)}(y) P_{y}\left(S_{\bar{\tau}_{A_{n}}}=x\right) .
$$

Then define

$$
\begin{aligned}
& \partial_{u}^{i n} \operatorname{Box}(n)=[-n, n] \times\left\{\left\lfloor n^{\alpha_{1}}\right\rfloor\right\} \\
& \partial_{d}^{i n} \operatorname{Box}(n)=[-n, n] \times\{0\} \\
& \partial_{l}^{i n} \operatorname{Box}(n)=\{-n\}, \times\left[1,\left\lfloor n^{\alpha_{1}}\right\rfloor-1\right] \\
& \partial_{r}^{i n} \operatorname{Box}(n)=\{n\}, \times\left[1,\left\lfloor n^{\alpha_{1}}\right\rfloor-1\right]
\end{aligned}
$$

to be the four edges of $\partial^{i n} \operatorname{Box}(n)$. Noting that $L_{0} \subset A$, it is easy to see that for any $y \in \partial_{d}^{i n} \operatorname{Box}(n)=$ $[-n, n] \times\{0\}, P_{y}\left(S_{\bar{\tau}_{A_{n}}}=x\right)=0$. Moreover, define $\alpha_{2}=(7+\alpha) / 8$ and

$$
l_{n}=\left[-\left\lfloor n^{\alpha_{2}}\right\rfloor,\left\lfloor n^{\alpha_{2}}\right\rfloor\right] \times\left\{\left\lfloor n^{\alpha_{1}}\right\rfloor\right\}
$$


to be the middle section of $\partial_{u}^{i n} \operatorname{Box}(n)$, and denote $l_{n}^{c}=\partial_{l}^{i n} \operatorname{Box}(n) \cup \partial_{r}^{i n} \operatorname{Box}(n) \cup \partial_{u}^{i n} \operatorname{Box}(n) \backslash l_{n}$. We further have the decomposition as follows:

$$
\mathcal{H}_{A_{n}}(x)=\sum_{y \in l_{n}^{c}} \mathcal{H}_{B o x(n)}(y) P_{y}\left(S_{\bar{\tau}_{A_{n}}}=x\right)+\sum_{y \in l_{n}} \mathcal{H}_{B o x(n)}(y) P_{y}\left(S_{\bar{\tau}_{A_{n}}}=x\right) .
$$

From (3.8), we first note that $\mathcal{H}_{B o x(n)}(y)$ sums up to 1 , which implies that

$$
\sum_{y \in l_{n}^{c}} \mathcal{H}_{B o x(n)}(y) P_{y}\left(S_{\bar{\tau}_{A_{n}}}=x\right) \leq \max _{y \in l_{n}^{c}} P_{y}\left(S_{\bar{\tau}_{A_{n}}}=x\right)
$$

Thus our first step is to prove

Proposition 3.6. For $\operatorname{Box}(n), l_{n}$, and $l_{n}^{c}$ defined as above, we have

$$
\lim _{n \rightarrow \infty} n \cdot \max _{y \in l_{n}^{c}} P_{y}\left(S \bar{\tau}_{A_{n}}=x\right)=0 .
$$

With Proposition 3.6, it is sufficient for us to concentrate on the asymptotic of

$$
\sum_{y \in l_{n}} \mathcal{H}_{B o x(n)}(y) P_{y}\left(S_{\bar{\tau}_{A_{n}}}=x\right)
$$

We are to show that

Proposition 3.7. For any $x \in A$ and the truncation $A_{n}$ defined in (1.1)

$$
\lim _{n \rightarrow \infty} \sum_{y \in l_{n}} P_{y}\left(S_{\bar{\tau}_{A_{n}}}=x\right)=\overline{\mathcal{H}}_{A}(x) .
$$

and that

Proposition 3.8. For any $\epsilon>0$, there is a $N_{0}<\infty$ such that for all $n \geq N_{0}$ and all $y \in l_{n}$,

$$
\left|2 \mathcal{H}_{\text {Box }(n)}(y)-\mathcal{H}_{D_{n}}(0)\right|<\epsilon / n \text {. }
$$

Once we have proved the lemmas above, Theorem 1.3 follows immediately from the combination of Propositions 3.6-3.8, together with Theorem 3.1.

3.3. Existence of upper and lower limit. In this subsection we prove Propositions 3.2 and 3.3. The following lemma can be easily obtained by using time reversibility and symmetry of simple random walk, e.g., see Lemma 2 of Kesten (1987).

Lemma 3.9. For any positive integer $n$ and $x \in D_{n}$,

$$
\mathcal{H}_{D_{n}}(x)=\lim _{R \rightarrow \infty} \frac{1}{\left|\partial^{\text {out }} B(0, R)\right|} E_{x}\left[\text { number of visits to } \partial^{\text {out }} B(0, R) \text { in }\left[0, \tau_{D_{n}}\right)\right] .
$$

The proofs of Propositions 3.2 and 3.3 rely on Lemma 3.9.

Proof of Proposition 3.2: By Lemma 3.9,

$$
\mathcal{H}_{D_{n}}(0)=\lim _{R \rightarrow \infty} \frac{1}{\left|\partial^{\text {out }} B(0, R)\right|} E_{0}\left[\text { number of visits to } \partial^{\text {out }} B(0, R) \text { in }\left[0, \tau_{D_{n}}\right)\right] .
$$

Note that there is a finite constant $C$ independent to $R$ such that

$$
\frac{1}{\left|\partial^{\text {out }} B(0, R)\right|} \leq \frac{C}{R}
$$


At the same time, define $C_{n}=[-\lfloor n / 2\rfloor, 0] \times\{0\} \subset D_{n}$ and apply Lemmas 3-4 of Kesten (1987) with $r=n$,

$$
\begin{aligned}
& E_{0}\left[\text { number of visits to } \partial^{\text {out }} B(0, R) \text { in }\left[0, \tau_{D_{n}}\right)\right] \\
& \leq \frac{P_{0}\left(\tau_{R}<\tau_{D_{n}}\right)}{\min _{w \in \partial^{\text {out }} B(0, R)} P_{w}\left(\tau_{D_{n}}<\tau_{R}\right)} \\
& \leq C R \log (R) P_{0}\left(\tau_{R}<\tau_{D_{n}}\right) \\
& =C R \log (R)\left(\sum_{z \in \partial^{\text {out }} B(0,2 n)} P_{0}\left(\tau_{2 n}<\tau_{D_{n}}, S_{\tau_{2 n}}=z\right) P_{z}\left(\tau_{R}<\tau_{D_{n}}\right)\right) \\
& \leq C R \log (R)\left(\sum_{z \in \partial^{\text {out }} B(0,2 n)} P_{0}\left(\tau_{2 n}<\tau_{D_{n}}, S_{\tau_{2 n}}=z\right) P_{z}\left(\tau_{R}<\tau_{C_{n}}\right)\right) \\
& \leq C R \log (R) P_{0}\left(\tau_{2 n}<\tau_{D_{n}}\right) \\
& \max _{z \in \partial^{\text {out }} B(0,2 n)} P_{z}\left(\tau_{R}<\tau_{C_{n}}\right) \\
& \leq C R P_{0}\left(\tau_{2 n}<\tau_{D_{n}}\right) .
\end{aligned}
$$

Thus, there is a finite constant $C$ independent to $n$ such that

$$
\mathcal{H}_{D_{n}}(x) \leq C P_{0}\left(\tau_{2 n}<\tau_{D_{n}}\right) .
$$

By Lemma 2.4 and the fact that $I_{n} \subset B(0,2 n)$, we have

$$
\begin{aligned}
P_{0}\left(\tau_{2 n}<\tau_{D_{n}}\right) & \leq P_{0}\left(\tau_{I_{n}}<\tau_{D_{n}}\right) \\
& =P_{0}\left(S_{T_{n}} \in L_{n} \cup \partial_{r}^{i n} I_{n} \cup \partial_{u}^{i n} I_{n}\right) \\
& \leq 2 P_{0}\left(S_{T_{n}} \in \partial_{u}^{i n} I_{n}\right) .
\end{aligned}
$$

Moreover, note that

$$
P_{0}\left(S_{T_{n}} \in \partial_{u}^{i n} I_{n}\right) \leq P_{0}\left(\tau_{L_{n}}<\tau_{L_{0}}\right)=\frac{1}{4 n}
$$

Combining (3.14), (3.15) and (3.16), the proof of Proposition 3.2 is complete.

The next lemma is an important ingredient in the proof of Proposition 3.3.

Lemma 3.10. For any $k \geq 2$, there is a $c_{k}>0$ such that

$$
P_{0}\left(\tau_{k n}<\tau_{D_{n}}\right) \geq \frac{c_{k}}{n} .
$$

Proof: For a simple random walk starting from 0 , it is easy to see that

$$
\tau_{k n} \leq \tau_{L_{k n}}, \tau_{L_{0}} \leq \tau_{D_{n}}
$$

Thus,

$$
P_{0}\left(\tau_{k n}<\tau_{D_{n}}\right) \geq P_{0}\left(\tau_{L_{k n}}<\tau_{L_{0}}\right)=\frac{1}{4 k n}
$$

and the proof of this lemma is complete.

Proof of Proposition 3.3: Recall (3.13). By Lemma 3.2 of Procaccia and Zhang (2019), there is a constant $C<\infty$ independent to the choice of $n$ and $R \gg n$ such that for all $w \in \partial^{\text {out }} B(0, R)$,

$$
P_{w}\left(\tau_{D_{n}}<\tau_{R}\right) \leq C[R \log (R)]^{-1} .
$$


Thus,

$$
\begin{aligned}
& E_{0}\left[\text { number of visits to } \partial^{\text {out }} B(0, R) \text { in }\left[0, \tau_{D_{n}}\right)\right] \\
& \geq \frac{P_{0}\left(\tau_{R}<\tau_{D_{n}}\right)}{\max _{w \in \partial^{\text {out }}} B(0, R)} P_{w}\left(\tau_{D_{n}}<\tau_{R}\right) \\
& \geq c R \log (R) P_{0}\left(\tau_{R}<\tau_{D_{n}}\right) .
\end{aligned}
$$

At the same time, by Lemma 3.3 of Procaccia and Zhang (2019), there are constants $2<c_{0}<\infty$ and $c>0$ independent to the choice of $n$ and $R \gg n$ such that for any $z \in \partial^{\text {out }} B\left(0, c_{0} n\right)$,

$$
P_{z}\left(\tau_{R}<\tau_{D_{n}}\right) \geq \frac{c}{\log (R)}
$$

Thus, we have

$$
\begin{aligned}
P_{0}\left(\tau_{R}<\tau_{D_{n}}\right) & =\sum_{z \in \partial^{\text {out }} B\left(0, c_{0} n\right)} P_{0}\left(\tau_{c_{0} n}<\tau_{D_{n}}, S_{\tau_{c_{0} n}}=z\right) P_{z}\left(\tau_{R}<\tau_{D_{n}}\right) \\
& \geq c R P_{0}\left(\tau_{c_{0} n}<\tau_{D_{n}}\right) .
\end{aligned}
$$

Therefore,

$$
\mathcal{H}_{D_{n}}(0) \geq c P_{0}\left(\tau_{c_{0} n}<\tau_{D_{n}}\right) .
$$

Combining (3.19) and Lemma 3.10, the proof is complete.

3.4. Proof of Proposition 3.4. For the proof of Proposition 3.4, we without loss of generality assume that the first coordinate of $x$ is an even number, see Remark 3.12 for details. With Propositions 3.2 and 3.3, by spatial translation it is easy to see that there are constants $0<c<C<\infty$ such that for all $x \in[-n / 2, n / 2]$,

Moreover, recall that

$$
\frac{c}{n}<\mathcal{H}_{D_{n}}(x)<\frac{C}{n}
$$

$$
\begin{aligned}
\mathcal{H}_{D_{n}}(x) & =\lim _{R \rightarrow \infty} \frac{1}{\left|\partial^{\text {out }} B(0, R)\right|} \sum_{y \in \partial^{\text {out }} B(0, R)} \mathcal{H}_{D_{n}}(y, x) \\
& =\lim _{R \rightarrow \infty} \frac{1}{\left|\partial^{\text {out }} B(0, R)\right|} E_{x}\left[\text { number of visits to } \partial^{\text {out }} B(0, R) \text { in }\left[0, \tau_{D_{n}}\right)\right] .
\end{aligned}
$$

Thus for any $n$ and $x$, there has to be a $R_{0}$ such that for all $R \geq R_{0}$,

$$
\mid \mathcal{H}_{D_{n}}(x)-\frac{1}{\left|\partial^{\text {out }} B(0, R)\right|} E_{x}\left[\text { number of visits to } \partial^{\text {out }} B(0, R) \text { in }\left[0, \tau_{D_{n}}\right)\right] \mid<\frac{\epsilon}{4 n}
$$

and

$$
\mid \mathcal{H}_{D_{n}}(0)-\frac{1}{\left|\partial^{\text {out }} B(0, R)\right|} E_{0}\left[\text { number of visits to } \partial^{\text {out }} B(0, R) \text { in }\left[0, \tau_{D_{n}}\right)\right] \mid<\frac{\epsilon}{4 n} \text {. }
$$

At the same time,

$$
\begin{aligned}
& E_{x}\left[\text { number of visits to } \partial^{\text {out }} B(0, R) \text { in }\left[0, \tau_{D_{n}}\right)\right] \\
= & \sum_{z \in \partial^{\text {out }} B(0,2 n)} P_{x}\left(\tau_{2 n}<\tau_{D_{n}}, S_{\tau_{2 n}}=z\right) \sum_{w \in \partial^{\text {out }} B(0, R)} \frac{P_{z}\left(\tau_{R}<\tau_{D_{n}}, S_{\tau_{R}}=w\right)}{P_{w}\left(\tau_{D_{n}}<\tau_{R}\right)}
\end{aligned}
$$

and

$$
\begin{aligned}
& E_{0}\left[\text { number of visits to } \partial^{\text {out }} B(0, R) \text { in }\left[0, \tau_{D_{n}}\right)\right] \\
= & \sum_{z \in \partial^{\text {out }} B(0,2 n)} P_{0}\left(\tau_{2 n}<\tau_{D_{n}}, S_{\tau_{2 n}}=\underset{w \in \partial^{\text {out }} B(0, R)}{z} \frac{P_{z}\left(\tau_{R}<\tau_{D_{n}}, S_{\tau_{R}}=w\right)}{P_{w}\left(\tau_{D_{n}}<\tau_{R}\right)} .\right.
\end{aligned}
$$


Thus we have

$$
\begin{gathered}
\left|\mathcal{H}_{D_{n}}(x)-\mathcal{H}_{D_{n}}(0)\right| \\
\leq \frac{1}{\left|\partial^{\text {out }} B(0, R)\right|} \sum_{z \in \partial^{\text {out }} B(0,2 n)}\left|P_{0}\left(\tau_{2 n}<\tau_{D_{n}}, S_{\tau_{2 n}}=z\right)-P_{x}\left(\tau_{2 n}<\tau_{D_{n}}, S_{\tau_{2 n}}=z\right)\right| \\
\quad\left(\sum_{w \in \partial^{\text {out }} B(0, R)} \frac{P_{z}\left(\tau_{R}<\tau_{D_{n}}, S_{\tau_{R}}=w\right)}{P_{w}\left(\tau_{D_{n}}<\tau_{R}\right)}\right)+\frac{\epsilon}{2 n} .
\end{gathered}
$$

Again by Lemmas 3-4 of Kesten (1987) with $r=n$, there is a constant $C<\infty$ such that for all $n$, $R \gg n$ and $z \in \partial^{\text {out }} B(0,2 n)$,

$$
\begin{aligned}
& \frac{1}{\left|\partial^{\text {out }} B(0, R)\right|}\left(\sum_{w \in \partial^{\text {out }} B(0, R)} \frac{P_{z}\left(\tau_{R}<\tau_{D_{n}}, S_{\tau_{R}}=w\right)}{P_{w}\left(\tau_{D_{n}}<\tau_{R}\right)}\right) \\
& \leq \frac{P_{z}\left(\tau_{R}<\tau_{D_{n}}\right)}{\left|\partial^{\text {out }} B(0, R)\right| \min _{w \in \partial^{\text {out }} B(0, R)} P_{w}\left(\tau_{D_{n}}<\tau_{R}\right)} \leq C .
\end{aligned}
$$

Thus by (3.21) and (3.22), in order to prove Proposition 3.4, it suffices to show the following lemma:

Lemma 3.11. For any $\epsilon>0$, there is a $\delta>0$ such that for all sufficiently large $n$ and any $x \in[-\delta n, \delta n] \times\{0\}$, we have

$$
\sum_{z \in \partial^{\text {out }} B(0,2 n)}\left|P_{0}\left(\tau_{2 n}<\tau_{D_{n}}, S_{\tau_{2 n}}=z\right)-P_{x}\left(\tau_{2 n}<\tau_{D_{n}}, S_{\tau_{2 n}}=z\right)\right|<\frac{\epsilon}{n} .
$$

Proof: For any $\epsilon>0$, define $\delta=e^{-\epsilon^{-1}}>0$. In order to prove this lemma, we construct the following coupling between the simple random walks starting from 0 and $x \in[-\delta n, \delta n] \times\{0\}$ :

(i) Define a subset $A_{n}^{\epsilon}=[-\lfloor n / 2\rfloor,\lfloor n / 2\rfloor] \times[0,\lfloor\epsilon n\rfloor]$.

(ii) Let $\left\{\bar{S}_{k}\right\}_{k=0}^{\infty}$ be a simple random walk starting from $0, \bar{T}_{n}^{\epsilon}=\inf \left\{k: \bar{S}_{k} \in \partial^{i n} A_{n}^{\epsilon}\right\}$, and $x_{n}^{\epsilon}=\bar{S}_{\bar{T}_{n}^{\epsilon}}$.

(iii) For $k \leq \bar{T}_{n}^{\epsilon}$, let $S_{1, k}=\bar{S}_{k}$ and $S_{2, k}=\bar{S}_{k}+x$.

(iv) Let $\left\{\hat{S}_{1, k}\right\}_{k=0}^{\infty}$ and $\left\{\hat{S}_{2, k}\right\}_{k=0}^{\infty}$ be two simple random walks starting from $x_{n}^{\epsilon}$ and $x_{n}^{\epsilon}+x$ and coupled under the maximal coupling.

(v) For $k>\bar{T}_{n}^{\epsilon}$, let $S_{1, k}=\hat{S}_{1, k-T_{n}^{\epsilon}}$ and $S_{2, k}=\hat{S}_{2, k-T_{n}^{\epsilon}}$.

Remark 3.12. In Step (iv) we use the assumption that the first coordinate of $x$ is an even number. Otherwise, one can construct $\hat{S}_{1, k}$ starting from $x_{n}^{\epsilon}$ and $\hat{S}_{2, k}$ starting uniformly from $B\left(x_{n}^{\epsilon}+x, 1\right)$ under maximal coupling.

By strong Markov property, it is easy to see that $S_{1, k}$ and $S_{2, k}$ form two simple random walks starting from 0 and $x$. Let $\tau^{(1)}$ and $\tau^{(2)}$ be the stopping times with respect to $S_{1, k}$ and $S_{2, k}$ respectively. Thus,

$$
\begin{aligned}
& \sum_{z \in \partial^{\text {out }} B(0,2 n)}\left|P_{0}\left(\tau_{2 n}<\tau_{D_{n}}, S_{\tau_{2 n}}=z\right)-P_{x}\left(\tau_{2 n}<\tau_{D_{n}}, S_{\tau_{2 n}}=z\right)\right| \\
= & \sum_{z \in \partial^{\text {out }} B(0,2 n)}\left|P_{0}\left(\tau_{2 n}^{(1)}<\tau_{D_{n}}^{(1)}, S_{1, \tau_{2 n}^{(1)}}=z\right)-P_{x}\left(\tau_{2 n}^{(2)}<\tau_{D_{n}}^{(2)}, S_{2, \tau_{2 n}^{(2)}}=z\right)\right| .
\end{aligned}
$$

Again we introduce

$$
U_{n}^{\epsilon}=[-\lfloor n / 2\rfloor,\lfloor n / 2\rfloor] \times\{\lfloor\epsilon n\rfloor\}, \quad B_{n}^{\epsilon}=[-\lfloor n / 2\rfloor,\lfloor n / 2\rfloor] \times\{0\}
$$

and

$$
L_{n}^{\epsilon}=\{-\lfloor n / 2\rfloor\} \times[1,\lfloor\epsilon n\rfloor-1], \quad R_{n}^{\epsilon}=\{\lfloor n / 2\rfloor\} \times[1,\lfloor\epsilon n\rfloor-1]
$$


as the four edges of $\partial^{i n} A_{n}^{\epsilon}$. Note that for all $\epsilon<1 / 3$,

$$
\left\{\tau_{2 n}^{(1)}<\tau_{D_{n}}^{(1)}\right\} \cap\left\{\bar{S}_{\bar{T}_{n}^{\epsilon}} \in B_{n}^{\epsilon}\right\}=\emptyset, \quad\left\{\tau_{2 n}^{(2)}<\tau_{D_{n}}^{(2)}\right\} \cap\left\{\bar{S}_{\bar{T}_{n}^{\epsilon}} \in B_{n}^{\epsilon}\right\}=\emptyset .
$$

Thus for any $z \in \partial^{\text {out }} B(0,2 n)$, we have

$$
\begin{aligned}
P_{0}\left(\tau_{2 n}^{(1)}<\tau_{D_{n}}^{(1)}, S_{1, \tau_{2 n}^{(1)}}=z\right) & =P_{0}\left(\bar{S}_{\bar{T}_{n}^{\epsilon}} \in U_{n}^{\epsilon}, \tau_{2 n}^{(1)}<\tau_{D_{n}}^{(1)}, S_{1, \tau_{2 n}^{(1)}}=z\right) \\
& +P_{0}\left(\bar{S}_{\bar{T}_{n}^{\epsilon}} \in L_{n}^{\epsilon} \cup R_{n}^{\epsilon}, \tau_{2 n}^{(1)}<\tau_{D_{n}}^{(1)}, S_{1, \tau_{2 n}^{(1)}}=z\right)
\end{aligned}
$$

and

$$
\begin{aligned}
P_{x}\left(\tau_{2 n}^{(2)}<\tau_{D_{n}}^{(2)}, S_{2, \tau_{2 n}^{(2)}}^{(2)}=z\right) & =P_{x}\left(\bar{S}_{\bar{T}_{n}^{\epsilon}} \in U_{n}^{\epsilon}, \tau_{2 n}^{(2)}<\tau_{D_{n}}^{(2)}, S_{2, \tau_{2 n}^{(2)}}=z\right) \\
& +P_{x}\left(\bar{S}_{\bar{T}_{n}^{\epsilon}} \in L_{n}^{\epsilon} \cup R_{n}^{\epsilon}, \tau_{2 n}^{(2)}<\tau_{D_{n}}^{(2)}, S_{2, \tau_{2 n}^{(2)}}^{(2)}=z\right) .
\end{aligned}
$$

Thus,

$$
\begin{aligned}
& \sum_{z \in \partial^{\text {out }} B(0,2 n)}\left|P_{0}\left(\tau_{2 n}^{(1)}<\tau_{D_{n}}^{(1)}, S_{1, \tau_{2 n}^{(1)}}=z\right)-P_{x}\left(\tau_{2 n}^{(2)}<\tau_{D_{n}}^{(2)}, S_{2, \tau_{2 n}^{(2)}}=z\right)\right| \\
\leq & \sum_{z \in \partial^{\text {out }} B(0,2 n)}\left|P\left(\bar{S}_{\bar{T}_{n}^{\epsilon}} \in U_{n}^{\epsilon}, \tau_{2 n}^{(1)}<\tau_{D_{n}}^{(1)}, S_{1, \tau_{2 n}^{(1)}}=z\right)-P\left(\bar{S}_{\bar{T}_{n}^{\epsilon}} \in U_{n}^{\epsilon}, \tau_{2 n}^{(2)}<\tau_{D_{n}}^{(2)}, S_{2, \tau_{2 n}^{(2)}}=z\right)\right| \\
+ & \sum_{z \in \partial^{\text {out }} B(0,2 n)} P\left(\bar{S}_{\bar{T}_{n}^{\epsilon}} \in L_{n}^{\epsilon} \cup R_{n}^{\epsilon}, \tau_{2 n}^{(1)}<\tau_{D_{n}}^{(1)}, S_{1, \tau_{2 n}^{(1)}}=z\right) \\
+ & \sum_{z \in \partial^{\text {out }} B(0,2 n)} P\left(\bar{S}_{\bar{T}_{n}^{\epsilon}} \in L_{n}^{\epsilon} \cup R_{n}^{\epsilon}, \tau_{2 n}^{(2)}<\tau_{D_{n}}^{(2)}, S_{2, \tau_{2 n}^{(2)}}^{(2)}=z\right) \\
\leq & \sum_{z \in \partial^{\text {out } B(0,2 n)}}\left|P\left(\bar{S}_{\bar{T}_{n}^{\epsilon}} \in U_{n}^{\epsilon}, \tau_{2 n}^{(1)}<\tau_{D_{n}}^{(1)}, S_{1, \tau_{2 n}^{(1)}}=z\right)-P\left(\bar{S}_{\bar{T}_{n}^{\epsilon}} \in U_{n}^{\epsilon}, \tau_{2 n}^{(2)}<\tau_{D_{n}}^{(2)}, S_{2, \tau_{2 n}^{(2)}}=z\right)\right| \\
+ & 2 P\left(\bar{S}_{\bar{T}_{n}^{\epsilon}} \in L_{n}^{\epsilon} \cup R_{n}^{\epsilon}\right) .
\end{aligned}
$$

In order to control the right hand side of (3.24), we first concentrate on controlling its second term. By invariance principle it is easy to check that there is a constant $c>0$ such that for any integer $m>1$ and any integer $j$ with $|j| \leq m$, we have

$$
P_{(0, j)}\left(\tau_{\partial_{l}^{i n} I_{m} \cup \partial_{r}^{i n} I_{m}}<\tau_{\partial_{u}^{i n} I_{m} \cup \partial_{b}^{i n} I_{m}}\right)<1-c .
$$

Moreover, by Lemma 2.4,

$$
P_{(0,0)}\left(\tau_{\partial_{l}^{i n} I_{m} \cup \partial_{r}^{i n} I_{m}}<\tau_{\partial_{u}^{i n} I_{m} \cup \partial_{b}^{i n} I_{m}}\right) \leq P_{(0,0)}\left(\tau_{L_{m}}<\tau_{L_{0}}\right)=\frac{1}{4 \epsilon m}
$$

In the rest of the proof we call the event in (3.25) a side escaping event. The detailed proof of (3.25) follows exactly the same argument as the proof of Equation (11) in Procaccia and Zhang (2021), which can also be illustrated in the following figure: 


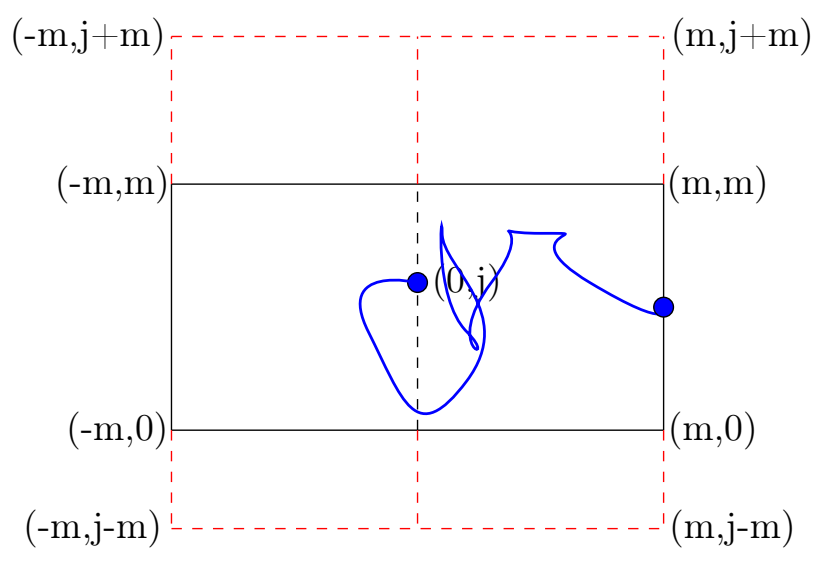

FIGURE 3.2. invariance principle for (3.25)

Moreover, define $m(\epsilon, n)=\lfloor\epsilon n\rfloor$. Note that in the event $\left\{\bar{S}_{\bar{T}_{n}^{\epsilon}} \in L_{n}^{\epsilon} \cup R_{n}^{\epsilon}\right\}$, our simple random walk has to first escape $A_{m(\epsilon, n)}$ through $L_{m(\epsilon, n)} \cup R_{m(\epsilon, n)}$ and then has at least $K(\epsilon, n)=\lfloor\lfloor n / 2\rfloor / m(\epsilon, n)\rfloor$ independent times of side escaping events. Thus by Lemma 2.4, (3.25), (3.26), and the fact that for all sufficiently small $\epsilon>0$,

we have

$$
K(\epsilon, n)=\lfloor\lfloor n / 2\rfloor / m(\epsilon, n)\rfloor \geq \frac{1}{3 \epsilon},
$$

$$
P\left(\bar{S}_{\bar{T}_{n}^{\epsilon}} \in L_{n}^{\epsilon} \cup R_{n}^{\epsilon}\right) \leq \frac{1}{4 \epsilon n}(1-c)^{\frac{1}{3 \epsilon}-1} \ll \frac{\epsilon}{n}
$$

for all sufficiently small $\epsilon>0$. Thus in order to prove Lemma 3.11, it suffices to show that

$$
\begin{aligned}
& \quad \sum_{z \in \partial^{\text {out }} B(0,2 n)}\left|P\left(\bar{S}_{\bar{T}_{n}^{\epsilon}} \in U_{n}^{\epsilon}, \tau_{2 n}^{(1)}<\tau_{D_{n}}^{(1)}, S_{1, \tau_{2 n}^{(1)}}=z\right)-P\left(\bar{S}_{\bar{T}_{n}^{\epsilon}} \in U_{n}^{\epsilon}, \tau_{2 n}^{(2)}<\tau_{D_{n}}^{(2)}, S_{2, \tau_{2 n}^{(2)}}=z\right)\right| \\
& \ll \frac{\epsilon}{n} .
\end{aligned}
$$

Recall that in our construction, $\left\{\hat{S}_{1, k}\right\}_{k=0}^{\infty}$ and $\left\{\hat{S}_{2, k}\right\}_{k=0}^{\infty}$ are simple random walks coupled under the maximal coupling. Define the events

and

$$
\begin{aligned}
& \mathcal{A}_{1}=\left\{\hat{S}_{1, k} \notin D_{n} \cup \partial^{\text {out }} B(0,2 n), \quad \forall k \leq \epsilon^{4} n^{2}\right\}, \\
& \mathcal{A}_{2}=\left\{\hat{S}_{2, k} \notin D_{n} \cup \partial^{\text {out }} B(0,2 n), \quad \forall k \leq \epsilon^{4} n^{2}\right\},
\end{aligned}
$$

$$
\mathcal{A}_{3}=\left\{\text { there exists a } k \leq \epsilon^{4} n^{2} \text { such that } \hat{S}_{1, j}=\hat{S}_{1, j}, \forall j \geq k\right\} .
$$

By definition, one can easily see that

$$
\begin{aligned}
& \left\{\bar{S}_{\bar{T}_{n}^{\epsilon}} \in U_{n}^{\epsilon}, \tau_{2 n}^{(1)}<\tau_{D_{n}}^{(1)}, S_{1, \tau_{2 n}^{(1)}}=z\right\} \cap \mathcal{A}_{1} \cap \mathcal{A}_{2} \cap \mathcal{A}_{3} \\
= & \left\{\bar{S}_{\bar{T}_{n}^{\epsilon}} \in U_{n}^{\epsilon}, \tau_{2 n}^{(2)}<\tau_{D_{n}}^{(2)}, S_{2, \tau_{2 n}^{(2)}}^{(2)}=z\right\} \cap \mathcal{A}_{1} \cap \mathcal{A}_{2} \cap \mathcal{A}_{3},
\end{aligned}
$$

which implies that

$$
\begin{aligned}
& \quad \sum_{z \in \partial^{\text {out }} B(0,2 n)}\left|P\left(\bar{S}_{\bar{T}_{n}^{\epsilon}} \in U_{n}^{\epsilon}, \tau_{2 n}^{(1)}<\tau_{D_{n}}^{(1)}, S_{1, \tau_{2 n}^{(1)}}=z\right)-P\left(\bar{S}_{\bar{T}_{n}^{\epsilon}} \in U_{n}^{\epsilon}, \tau_{2 n}^{(2)}<\tau_{D_{n}}^{(2)}, S_{2, \tau_{2 n}^{(2)}}=z\right)\right| \\
& \leq 2 P\left(\left\{\bar{S}_{\bar{T}_{n}^{\epsilon}} \in U_{n}^{\epsilon}\right\} \cap \mathcal{A}_{1}^{c}\right)+2 P\left(\left\{\bar{S}_{\bar{T}_{n}^{\epsilon}} \in U_{n}^{\epsilon}\right\} \cap \mathcal{A}_{2}^{c}\right)+2 P\left(\left\{\bar{S}_{\bar{T}_{n}^{\epsilon}} \in U_{n}^{\epsilon}\right\} \cap \mathcal{A}_{3}^{c}\right) .
\end{aligned}
$$


Thus, it suffices to control the probabilities on the right hand side of (3.30). For its first term, by Proposition 2.1.2 of Lawler and Limic (2010) there are constants $c, \beta \in(0, \infty)$ independent to $n$ such that

$$
P\left(\mathcal{A}_{1}^{c}\right) \leq c e^{-\beta / \epsilon^{2}}, \quad P\left(\mathcal{A}_{2}^{c}\right) \leq c e^{-\beta / \epsilon^{2}} .
$$

By strong Markov property, we have

$$
P\left(\left\{\bar{S}_{\bar{T}_{n}^{\epsilon}} \in U_{n}^{\epsilon}\right\} \cap \mathcal{A}_{1}^{c}\right) \leq \frac{c e^{-\beta / \epsilon^{2}}}{\epsilon} n^{-1} \ll \frac{\epsilon}{n}
$$

and

$$
P\left(\left\{\bar{S}_{\bar{T}_{n}^{\epsilon}} \in U_{n}^{\epsilon}\right\} \cap \mathcal{A}_{2}^{c}\right) \leq \frac{c e^{-\beta / \epsilon^{2}}}{\epsilon} n^{-1} \ll \frac{\epsilon}{n}
$$

for all sufficiently small $\epsilon>0$. Finally, for the last term

$$
P\left(\left\{\bar{S}_{\bar{T}_{n}^{\epsilon}} \in U_{n}^{\epsilon}\right\} \cap \mathcal{A}_{3}^{c}\right),
$$

recall that the first coordinate of $x$ is even and that $\left\{\hat{S}_{1, k}\right\}_{k=0}^{\infty}$ and $\left\{\hat{S}_{2, k}\right\}_{k=0}^{\infty}$ are two simple random walks starting from $x_{n}^{\epsilon}$ and $x_{n}^{\epsilon}+x$ and coupled under the maximal coupling. We have

$$
P\left(\mathcal{A}_{3}^{c}\right) \leq d_{T V}\left(\hat{S}_{1,\left\lfloor\epsilon^{4} n^{2}\right\rfloor}, \hat{S}_{2,\left\lfloor\epsilon^{4} n^{2}\right\rfloor}\right)
$$

where $d_{T V}(\cdot, \cdot)$ stands for the total variation distance between the distributions of two random variables. On the other hand, note that

$$
\begin{aligned}
d_{T V}\left(\hat{S}_{1,\left\lfloor\epsilon^{4} n^{2}\right\rfloor}, \hat{S}_{2,\left\lfloor\epsilon^{4} n^{2}\right\rfloor}\right) & =\frac{1}{2} \sum_{z \in \mathbb{Z}^{2}}\left|P\left(\hat{S}_{1,\left\lfloor\epsilon^{4} n^{2}\right\rfloor}=z\right)-P\left(\hat{S}_{2,\left\lfloor\epsilon^{4} n^{2}\right\rfloor}=z\right)\right| \\
& \leq \frac{1}{2}\left[P\left(\hat{S}_{1,\left\lfloor\epsilon^{4} n^{2}\right\rfloor} \in B^{c}(0,2 n)\right)+P\left(\hat{S}_{2,\left\lfloor\epsilon^{4} n^{2}\right\rfloor} \in B^{c}(0,2 n)\right)\right. \\
& \left.+\sum_{z \in B(0,2 n)}\left|P\left(\hat{S}_{1,\left\lfloor\epsilon^{4} n^{2}\right\rfloor}=z\right)-P\left(\hat{S}_{2,\left\lfloor\epsilon^{4} n^{2}\right\rfloor}=z\right)\right|\right] .
\end{aligned}
$$

And again by Proposition 2.1.2 of Lawler and Limic (2010), there are constants $c, \beta \in(0, \infty)$ independent to $n$ such that

$$
P\left(\hat{S}_{1,\left\lfloor\epsilon^{4} n^{2}\right\rfloor} \in B^{c}(0,2 n)\right) \leq c e^{-\beta / \epsilon^{4}}, P\left(\hat{S}_{2,\left\lfloor\epsilon^{4} n^{2}\right\rfloor} \in B^{c}(0,2 n)\right) \leq c e^{-\beta / \epsilon^{4}} .
$$

And for any $z \in B(0,2 n)$, conditioning on the event $\left\{\bar{S}_{\bar{T}_{n}^{\epsilon}}=x_{n}^{\epsilon}\right\}$ and applying Proposition 4.1 of Delmotte (1999) with $x_{0}=x_{n}^{\epsilon}, n_{0}=\left\lfloor\epsilon^{4} n^{2}\right\rfloor$ and $R=\left\lfloor\epsilon^{4} n\right\rfloor$, there are constant $h>0$ and $C<\infty$ independent to $n$ and $x_{n}^{\epsilon}$ such that

$$
\begin{aligned}
& \left|P\left(\hat{S}_{1,\left\lfloor\epsilon^{4} n^{2}\right\rfloor}=z \mid \bar{S}_{\bar{T}_{n}^{\epsilon}}=x_{n}^{\epsilon}\right)-P\left(\hat{S}_{2,\left\lfloor\epsilon^{4} n^{2}\right\rfloor}=z \mid \bar{S}_{\bar{T}_{n}^{\epsilon}}=x_{n}^{\epsilon}\right)\right| \\
& \leq C\left(\frac{e^{-\frac{1}{\epsilon}}}{\epsilon^{4}}\right)^{h} \sup _{(n, y) \in Q} P_{y}\left(S_{n}=z\right)
\end{aligned}
$$

where $Q=\left[n_{0}-2 R^{2}, n_{0}\right] \times B\left(x_{n}^{\epsilon}, 2 R\right)$. Moreover, by Local Central Limit Theorem, see Theorem 2.1.1 of Lawler and Limic (2010) for example, there is a finite constant $C<\infty$ independent to $n$ such that

$$
\sup _{(n, y) \in Q} P_{y}\left(S_{n}=z\right) \leq \frac{C}{\epsilon^{4} n^{2}}
$$


which implies that

$$
\left(\frac{e^{-\frac{1}{\epsilon}}}{\epsilon^{4}}\right)^{h} \sup _{(n, y) \in Q} P_{y}\left(S_{n}=z\right) \leq C e^{-\frac{h}{\epsilon}} \epsilon^{-4(1+h)} n^{-2}
$$

and that

$$
\begin{aligned}
& \left|P\left(\hat{S}_{1,\left\lfloor\epsilon^{4} n^{2}\right\rfloor}=z\right)-P\left(\hat{S}_{2,\left\lfloor\epsilon^{4} n^{2}\right\rfloor}=z\right)\right| \\
& \leq \sum_{x_{n}^{\epsilon}}\left|P\left(\hat{S}_{1,\left\lfloor\epsilon^{4} n^{2}\right\rfloor}=z \mid \bar{S}_{\bar{T}_{n}^{\epsilon}}=x_{n}^{\epsilon}\right)-P\left(\hat{S}_{2,\left\lfloor\epsilon^{4} n^{2}\right\rfloor}=z \mid \bar{S}_{\bar{T}_{n}^{\epsilon}}=x_{n}^{\epsilon}\right)\right| P\left(\bar{S}_{\bar{T}_{n}^{\epsilon}}=x_{n}^{\epsilon}\right) \\
& \leq C e^{-\frac{h}{\epsilon}} \epsilon^{-4(1+h)} n^{-2} \sum_{x_{n}^{\epsilon}} P\left(\bar{S}_{\bar{T}_{n}^{\epsilon}}=x_{n}^{\epsilon}\right) \\
& \leq C e^{-\frac{h}{\epsilon}} \epsilon^{-4(1+h)} n^{-2} .
\end{aligned}
$$

Thus,

$$
\begin{aligned}
& \sum_{z \in B(0,2 n)}\left|P\left(\hat{S}_{1,\left\lfloor\epsilon^{4} n^{2}\right\rfloor}=z\right)-P\left(\hat{S}_{2,\left\lfloor\epsilon^{4} n^{2}\right\rfloor}=z\right)\right| \\
\leq & \sum_{z \in B(0,2 n)} C e^{-\frac{h}{\epsilon}} \epsilon^{-4(1+h)} n^{-2} \\
\leq & C e^{-\frac{h}{\epsilon}} \epsilon^{-4(1+h)} .
\end{aligned}
$$

Combining (3.33) and (3.35), we have

$$
P\left(\mathcal{A}_{3}^{c}\right) \leq d_{T V}\left(\hat{S}_{1,\left\lfloor\epsilon^{4} n^{2}\right\rfloor}, \hat{S}_{2,\left\lfloor\epsilon^{4} n^{2}\right\rfloor}\right) \leq \frac{1}{2}\left(2 c e^{-\beta / \epsilon^{4}}+C e^{-\frac{h}{\epsilon}} \epsilon^{-4(1+h)}\right) .
$$

And by strong Markov property,

$$
P\left(\left\{\bar{S}_{\bar{T}_{n}^{\epsilon}} \in U_{n}^{\epsilon}\right\} \cap \mathcal{A}_{3}^{c}\right) \leq \frac{1}{8 \epsilon n}\left(2 c e^{-\beta / \epsilon^{4}}+C e^{-\frac{h}{\epsilon}} \epsilon^{-4(1+h)}\right) \ll \frac{\epsilon}{n}
$$

for all sufficiently large $n$ and sufficiently small $\epsilon$. Thus, the proof of this lemma is complete.

With Lemma 3.11, the proof of Proposition 3.4 is complete.

3.5. Proof of Lemma 3.5. Let $M, M_{0} \in \mathbb{Z}_{+}$such that $M>M_{0}>1$. By strong Markov property,

$$
\begin{aligned}
& P_{(0, M n)}\left(\tau_{[-\delta n, \delta n] \times\{0\}}=\tau_{D_{n}}\right) \\
& =\sum_{y \in \partial^{\text {out }} B\left(0, M_{0} n\right)} P_{(0, M n)}\left(\tau_{\partial^{\text {out }} B\left(0, M_{0} n\right)}=y\right) P_{y}\left(\tau_{[-\delta n, \delta n] \times\{0\}}=\tau_{D_{n}}\right) .
\end{aligned}
$$

By law of total probability,

$$
\begin{aligned}
& \min _{y \in \partial^{\text {out }} B\left(0, M_{0} n\right)} P_{y}\left(\tau_{[-\delta n, \delta n] \times\{0\}}=\tau_{D_{n}}\right) \\
& \leq P_{(0, M n)}\left(\tau_{[-\delta n, \delta n] \times\{0\}}=\tau_{D_{n}}\right) \\
& \leq \max _{y \in \partial^{\text {out }} B\left(0, M_{0} n\right)} P_{y}\left(\tau_{[-\delta n, \delta n] \times\{0\}}=\tau_{D_{n}}\right) .
\end{aligned}
$$

Notice that if we fix $n$,

$$
\lim _{M \rightarrow \infty} P_{(0, M n)}\left(\tau_{[-\delta n, \delta n] \times\{0\}}=\tau_{D_{n}}\right)=\mathcal{H}_{D_{n}}([-\delta n, \delta n] \times\{0\}),
$$


and thus

$$
\begin{aligned}
& \min _{y \in \partial^{\text {out }} B\left(0, M_{0} n\right)} P_{y}\left(\tau_{[-\delta n, \delta n] \times\{0\}}=\tau_{D_{n}}\right) \\
& \leq \mathcal{H}_{D_{n}}([-\delta n, \delta n] \times\{0\}) \\
& \leq \max _{y \in \partial^{\text {out }} B\left(0, M_{0} n\right)} P_{y}\left(\tau_{[-\delta n, \delta n] \times\{0\}}=\tau_{D_{n}}\right) .
\end{aligned}
$$

Let $\left\{y_{n}: y_{n} \in \partial^{\text {out }} B\left(0, M_{0} n\right)\right\}$ be a sequence of points in $\mathbb{Z}^{2}$. Note that $\left\|y_{n}\right\|_{2} \rightarrow \infty$ as $n \rightarrow \infty$. By invariance principle,

$$
\limsup _{n \rightarrow \infty} P_{y_{n}}\left(\tau_{[-\delta n, \delta n] \times\{0\}}=\tau_{D_{n}}\right) \leq \sup _{z \in \partial \bar{B}\left(0, M_{0}\right)} P_{z}^{B M}\left(\tau_{[-\delta, \delta] \times\{0\}}=\tau_{[-1,1] \times\{0\}}\right),
$$

where $P_{z}^{B M}$ is the law of a Brownian motion starting at the point $z \in \mathbb{R}^{2}$. Since the choice of $\left\{y_{n}\right\}$ is arbitrary,

$$
\limsup _{n \rightarrow \infty} \max _{y \in \partial^{\text {out }} B\left(0, M_{0} n\right)} P_{y}\left(\tau_{[-\delta n, \delta n] \times\{0\}}=\tau_{D_{n}}\right) \leq \sup _{z \in \partial \bar{B}\left(0, M_{0}\right)} P_{z}^{B M}\left(\tau_{[-\delta, \delta] \times\{0\}}=\tau_{[-1,1] \times\{0\}}\right) .
$$

Similarly,

$$
\liminf _{n \rightarrow \infty} \min _{y \in \partial^{\text {out }} B\left(0, M_{0} n\right)} P_{y}\left(\tau_{[-\delta n, \delta n] \times\{0\}}=\tau_{D_{n}}\right) \geq \inf _{z \in \partial \bar{B}\left(0, M_{0}\right)} P_{z}^{B M}\left(\tau_{[-\delta, \delta] \times\{0\}}=\tau_{[-1,1] \times\{0\}}\right) .
$$

Note that

$$
\begin{aligned}
& \lim _{M_{0} \rightarrow \infty} \sup _{z \in \partial \bar{B}\left(0, M_{0}\right)} P_{z}^{B M}\left(\tau_{[-\delta, \delta] \times\{0\}}=\tau_{[-1,1] \times\{0\}}\right) \\
& =\lim _{M_{0} \rightarrow \infty} \inf _{z \in \partial \bar{B}\left(0, M_{0}\right)} P_{z}^{B M}\left(\tau_{[-\delta, \delta] \times\{0\}}=\tau_{[-1,1] \times\{0\}}\right) \\
& =\mathrm{H}_{[-1,1] \times\{0\}}([-\delta, \delta] \times\{0\}) .
\end{aligned}
$$

Therefore,

$$
\lim _{n \rightarrow \infty} \mathcal{H}_{D_{n}}([-\delta n, \delta n] \times\{0\})=\mathrm{H}_{[-1,1] \times\{0\}}([-\delta, \delta] \times\{0\})
$$

With Lemma 3.5, the proof of Theorem 3.1 is complete.

3.6. Proof of Proposition 3.6. In order to prove

$$
\lim _{n \rightarrow \infty} n \cdot \max _{y \in l_{n}^{c}} P_{y}\left(S_{\bar{\tau}_{A_{n}}}=x\right)=0
$$

we first recall that

$$
\begin{gathered}
l_{n}=\left[-\left\lfloor n^{\alpha_{2}}\right\rfloor,\left\lfloor n^{\alpha_{2}}\right\rfloor\right] \times\left\{\left\lfloor n^{\alpha_{1}}\right\rfloor\right\}, \\
\alpha_{1}=(1+\alpha) / 2, \\
\alpha_{2}=(7+\alpha) / 8,
\end{gathered}
$$

and that

$$
l_{n}^{c}=\partial_{l}^{i n} \operatorname{Box}(n) \cup \partial_{r}^{i n} \operatorname{Box}(n) \cup \partial_{u}^{i n} \operatorname{Box}(n) \backslash l_{n} .
$$

Thus for any point $y \in l_{n}^{c}$, define

$$
T_{y}=\left\{\left\lfloor y^{(1)} / 2\right\rfloor\right\} \times[0, \infty)
$$


to be the vertical line located in the exact midway between 0 and $y$. Noting that $\tau_{T_{y}}<\tau_{x}$, by strong Markov property we have

$$
\begin{aligned}
P_{y}\left(S_{\bar{\tau}_{A_{n}}}=x\right) & =\sum_{z \in T_{y}} P_{y}\left(\tau_{T_{y}}<\bar{\tau}_{A_{n}}, S_{\tau_{T_{y}}}=z\right) P_{z}\left(S_{\bar{\tau}_{A_{n}}}=x\right) \\
& =\sum_{z \in T_{y}, z^{(2)} \geq n^{4}} P_{y}\left(\tau_{T_{y}}<\bar{\tau}_{A_{n}}, S_{\tau_{T_{y}}}=z\right) P_{z}\left(S_{\bar{\tau}_{A_{n}}}=x\right) \\
& +\sum_{z \in T_{y}, z^{(2)}<n^{4}} P_{y}\left(\tau_{T_{y}}<\bar{\tau}_{A_{n}}, S_{\tau_{T_{y}}}=z\right) P_{z}\left(S_{\bar{\tau}_{A_{n}}}=x\right) \\
& \leq P_{y}\left(\tau_{T_{y}}<\bar{\tau}_{A_{n}}, S_{T_{y}}^{(2)} \geq n^{4}\right) \\
& \left.+\max _{z \in T_{y}, z^{(2)}<n^{4}} P_{\bar{\tau}_{A_{n}}}=x\right) P_{y}\left(\tau_{T_{y}}<\bar{\tau}_{A_{n}}\right) .
\end{aligned}
$$

To control the right hand side of (3.42), we first define

$$
\bar{D}_{n}=\left\{T_{y} \cup[\lfloor y / 2\rfloor, \infty) \times\{0\}\right\} \cap B\left(y, n^{4}\right)
$$

and then note that

$$
P_{y}\left(\tau_{T_{y}}<\bar{\tau}_{A_{n}}, S_{T_{y}}^{(2)} \geq n^{4}\right) \leq P_{y}\left(\tau_{\partial^{\text {out }} B\left(y, n^{4}\right)}<\tau_{\bar{D}_{n}}\right)
$$

Moreover, it is easy to see that

$$
\operatorname{rad}\left(\bar{D}_{n}\right) \geq n^{4} / 2
$$

for $n$ sufficiently large, and that

$$
d\left(\bar{D}_{n}, y\right) \leq\left\lfloor n^{\alpha_{1}}\right\rfloor .
$$

We apply Theorem 1 in Lawler and Limic (2004) with $\kappa=1$ and $A=\bar{D}_{n}$ on the discrete ball $B\left(y, n^{4}\right)$, so there exists a constant $C>0$ such that

$$
P_{y}\left(\tau_{\partial^{\text {out }} B\left(y, n^{4}\right)}<\tau_{\bar{D}_{n}}\right) \leq P_{y}\left(\tau_{\partial^{\text {out }} B\left(y, n^{4}\right)}<\tau_{\bar{D}_{n_{\left[n^{\alpha}, n^{4} / 2\right]}}}\right) \leq C \sqrt{\frac{n^{\alpha_{1}}}{n^{4}}}=o\left(\frac{1}{n}\right) .
$$

Note that this is a Beurling estimate for random walk. And for the second term in the right hand side of (3.42), note that for

$$
\tilde{D}_{n}=L_{0} \cap B\left(y, n^{\alpha_{2}} / 2\right)
$$

we have

$$
\left\{\tau_{T_{y}}<\bar{\tau}_{A_{n}}\right\} \subset\left\{\tau_{\partial^{o u t} B\left(y, n^{\alpha} / 2\right)}<\bar{\tau}_{\tilde{D}_{n}}\right\}
$$

Using Theorem 1 of Lawler and Limic (2004) again to the right hand side of (3.44), we have

$$
P_{y}\left(\tau_{T_{y}}<\bar{\tau}_{A_{n}}\right) \leq P_{y}\left(\tau_{\partial^{\text {out }} B\left(y, n^{\alpha} / 2\right)}<\bar{\tau}_{\tilde{D}_{n}}\right) \leq C n^{-\left(\alpha_{2}-\alpha_{1}\right) / 2} .
$$


At the same time, for any $z \in T_{y}$ such that $z^{(2)}<n^{4}$, again by the reversibility of simple random walk we have

$$
\begin{aligned}
P_{z}\left(S_{\bar{\tau}_{A_{n}}}=x\right) & =\sum_{n=1}^{\infty} P_{z}\left(S_{1}, S_{2}, \cdots, S_{n-1} \notin A_{n}, S_{n}=x\right) \\
& =\sum_{n=1}^{\infty} P_{x}\left(S_{1}, S_{2}, \cdots, S_{n-1} \notin A_{n}, S_{n}=z\right) \\
& =E_{x}\left[\# \text { of visits to } z \text { in }\left[0, \tau_{A_{n}}\right)\right] \\
& =P_{x}\left(\tau_{z}<\tau_{A_{n}}\right) E_{z}\left[\# \text { of visits to } z \text { in }\left[0, \tau_{A_{n}}\right)\right] \\
& =\frac{P_{x}\left(\tau_{z}<\tau_{A_{n}}\right)}{P_{z}\left(\tau_{A_{n}}<\tau_{z}\right)} .
\end{aligned}
$$

To control the right hand side of (3.46), we first refer to the following well known result:

Lemma 3.13. (Lemma 1 of Kesten (1987)) The series

$$
a(x)=\sum_{n=0}^{\infty}\left[P_{0}\left(S_{n}=0\right)-P_{0}\left(S_{n}=x\right)\right]
$$

converge for each $x \in \mathbb{Z}^{2}$, and the function $a(\cdot)$ has the following properties:

$$
\begin{gathered}
a(x) \geq 0, \forall x \in \mathbb{Z}^{2}, a(0)=0, \\
a(( \pm 1,0))=a((0, \pm 1))=1 \\
E_{x}\left[a\left(S_{1}\right)\right]-a(x)=\delta(x, 0),
\end{gathered}
$$

so $a\left(S_{n \wedge \tau_{v}}-v\right)$ is a nonnegative martingale, where $\tau_{v}=\tau_{\{v\}}$, for any $v \in \mathbb{Z}^{2}$. And there is some suitable $c_{0}$ such that

$$
\left|a(x)-\frac{1}{2 \pi} \log \|x\|-c_{0}\right|=O\left(\|x\|^{-2}\right),
$$

as $\|x\| \rightarrow \infty$.

Now we prove the following lower bound on the denominator:

Lemma 3.14. There is a finite constant $C<\infty$ such that for any nonzero $x \in \mathbb{Z}^{2}$,

$$
P_{0}\left(\tau_{x}<\tau_{0}\right) \geq \frac{C}{(\log \|x\|)^{2}}
$$

Proof: First, it suffices to show this lemma for all $x$ sufficiently far away from 0 . We consider the stopping time

$$
\Gamma=\tau_{0} \wedge \tau_{\|x\| / 2}
$$

By Lemma 3.13, we have

$$
1=E_{0}\left[a\left(S_{\Gamma}\right) \mid \tau_{\|x\| / 2}<\tau_{0}\right] P_{0}\left(\tau_{\|x\| / 2}<\tau_{0}\right) .
$$

Thus by (3.51),

$$
P_{0}\left(\tau_{\|x\| / 2}<\tau_{0}\right)=\frac{1}{E_{0}\left[a\left(S_{\Gamma}\right) \mid \tau_{\|x\| / 2}<\tau_{0}\right]} \geq \frac{\pi}{\log \|x\|}
$$


for all $x$ sufficiently far away from 0. By strong Markov property,

$$
\begin{aligned}
P_{0}\left(\tau_{x}<\tau_{0}\right) & =\sum_{y \in \partial^{\text {out }} B(0,\|x\| / 2)} P_{0}\left(\tau_{\|x\| / 2}<\tau_{0}, S_{\tau_{\|x\| / 2}}=y\right) P_{y}\left(\tau_{x}<\tau_{0}\right) \\
& \geq \frac{\pi}{\log \|x\|} \min _{y \in \partial^{\text {out }} B(0,\|x\| / 2)} P_{y}\left(\tau_{x}<\tau_{0}\right) .
\end{aligned}
$$

At the same time, for stopping times $\Gamma_{1}=\tau_{\partial^{\text {out }}} B(x,\|x\| / 3)$ and $\Gamma_{2}=\tau_{\partial^{\text {out }}} B(x,\|x\| / 2)$, we have

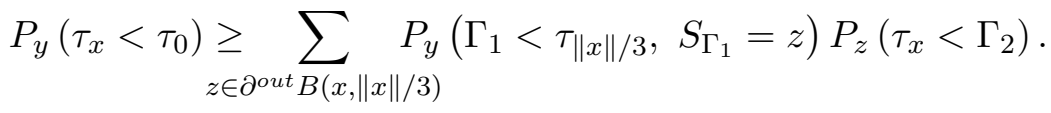

For the right hand side of (3.54), we have by translation invariance of simple random walk,

$$
P_{z}\left(\tau_{x}<\Gamma_{2}\right)=P_{z-x}\left(\tau_{0}<\tau_{\|x\| / 2}\right) .
$$

Moreover,

$$
\left[1-P_{z-x}\left(\tau_{0}<\tau_{\|x\| / 2}\right)\right] E_{z-x}\left[\left.a\left(S_{\Gamma}\right)\right|_{\|x\| / 2}<\tau_{0}\right]=a(z-x),
$$

which implies that

$$
P_{z-x}\left(\tau_{0}<\tau_{\|x\| / 2}\right)=\frac{E_{z-x}\left[a\left(S_{\Gamma}\right) \mid \tau_{\|x\| / 2}<\tau_{0}\right]-a(z-x)}{E_{z-x}\left[a\left(S_{\Gamma}\right) \mid \tau_{\|x\| / 2}<\tau_{0}\right]} .
$$

Again, by Lemma 3.13, there are positive constants $c, C \in(0, \infty)$ such that uniformly for all $n, x$ and $z$ defined above,

$$
E_{z-x}\left[a\left(S_{\Gamma}\right) \mid \tau_{\|x\| / 2}<\tau_{0}\right]-a(z-x) \geq c,
$$

while

$$
E_{z-x}\left[a\left(S_{\Gamma}\right) \mid \tau_{\|x\| / 2}<\tau_{0}\right] \leq C \log \|x\| .
$$

Thus, we have

$$
P_{z}\left(\tau_{x}<\Gamma_{2}\right)=P_{z-x}\left(\tau_{0}<\tau_{\|x\| / 2}\right) \geq \frac{c}{\log \|x\|}
$$

uniformly for all $n, x$ and $z$ defined above.

On the other hand, by invariance principle, there is a constant $c>0$ such that for any $y \in$ $\partial^{\text {out }} B(0,\|x\| / 2)$,

Thus,

$$
P_{y}\left(\Gamma_{1}<\tau_{\|x\| / 3}\right) \geq c .
$$

$$
P_{y}\left(\tau_{x}<\tau_{0}\right) \geq \sum_{z \in \partial^{\text {out }} B(x,\|x\| / 3)} P_{y}\left(\Gamma_{1}<\tau_{\|x\| / 3}, X_{\Gamma_{1}}=z\right) P_{z}\left(\tau_{x}<\Gamma_{2}\right) \geq \frac{c}{\log \|x\|} .
$$

Now combining (3.52), (3.53), and (3.57), the proof of this lemma is complete.

With Lemma 3.14, we look back at the right hand side of (3.46). Noting that for any $z \in T_{y}$, $\tau_{T_{y}} \leq \tau_{z}$ and that $\tau_{A_{n}} \leq \tau_{D_{n}}$, we give the following upper bound estimate on its numerator:

Lemma 3.15. Recall that $\alpha_{2}=(7+\alpha) / 8$. For each $x \in A$,

$$
P_{x}\left(\tau_{T_{y}}<\tau_{D_{n}}\right) \leq \frac{c}{n^{\alpha_{2}}}
$$

for all sufficiently large $n$ and all $y \in l_{n}^{c}$.

Proof: For any given $x \in A$, define $x_{0}=\left(x^{(1)}, 0\right)$ be the projection of $x$ on $L_{0}$. Note that $x_{0}$ and $x$ are connected by a path independent to $n$, which implies that there is a constant $c>0$ also independent to $n$ such that

$$
P_{x_{0}}\left(\tau_{T_{y}}<\tau_{D_{n}}\right) \geq c P_{x}\left(\tau_{T_{y}}<\tau_{D_{n}}\right)
$$


Thus to prove Lemma 3.15, it suffices to replace $x$ by $x_{0}$. Moreover, recall that $l_{n}^{c}=\partial_{l}^{i n} B o x(n) \cup$ $\partial_{r}^{i n} \operatorname{Box}(n) \cup \partial_{u}^{i n} \operatorname{Box}(n) \backslash l_{n}$. For any $y \in l_{n}^{c}$, by the translation invariance of simple random walk, we have

$$
P_{x_{0}}\left(\tau_{T_{y}}<\tau_{D_{n}}\right) \leq P_{0}\left(\tau_{I_{\left\lfloor n^{\alpha} / 4\right\rfloor}}<\tau_{D_{n}}\right) .
$$

Here recall the definition of $I_{n}$ in (2.4). Now by lemma 2.4,

$$
P_{0}\left(\tau_{I_{\left\lfloor n^{\alpha} / 4\right\rfloor}}<\tau_{D_{n}}\right) \leq \frac{C}{\left\lfloor n^{\left.\alpha_{2} / 4\right\rfloor}\right.}
$$

and the proof of this lemma is complete.

Now apply (3.45), (3.46), Lemma 3.14, and Lemma 3.15 together to the last term of (3.42), we have

$$
\begin{aligned}
\max _{z \in T_{y}, z^{(2)}<n^{4}} P_{z}\left(S_{\bar{\tau}_{A_{n}}}=x\right) P_{y}\left(\tau_{T_{y}}<\bar{\tau}_{A_{n}}\right) & \leq C n^{-\alpha_{2}-\left(\alpha_{2}-\alpha_{1}\right) / 2}(\log n)^{2} \\
& \leq C n^{-\frac{17}{16}+\frac{\alpha}{16}}(\log n)^{2} \ll n^{-1}
\end{aligned}
$$

for all sufficiently large $n$. Thus, the proof of Proposition 3.6 is complete.

3.7. Proof of Proposition 3.7. To show

$$
\lim _{n \rightarrow \infty} \sum_{y \in l_{n}} P_{y}\left(S_{\bar{\tau}_{A_{n}}}=x\right)=\overline{\mathcal{H}}_{A}(x)
$$

we first prove that

Lemma 3.16. For any $x \in A$ and the truncations $A_{n}$ defined in (1.1)

$$
\lim _{n \rightarrow \infty} \sum_{y \in l_{n}} P_{y}\left(S_{\bar{\tau}_{A}}=x\right)=\overline{\mathcal{H}}_{A}(x) .
$$

Proof: Recall that by definition

$$
\overline{\mathcal{H}}_{A}(x)=\lim _{k \rightarrow \infty} \sum_{z \in L_{k}} P_{z}\left(S_{\bar{\tau}_{A}}=x\right)
$$

and

Thus,

$$
l_{n}=\left[-\left\lfloor n^{\alpha_{2}}\right\rfloor,\left\lfloor n^{\alpha_{2}}\right\rfloor\right] \times\left\{\left\lfloor n^{\alpha_{1}}\right\rfloor\right\}
$$

$$
\lim _{n \rightarrow \infty} \sum_{z \in L_{\left\lfloor n^{\alpha}\right\rfloor}} P_{z}\left(S_{\bar{\tau}_{A}}=x\right)=\overline{\mathcal{H}}_{A}(x),
$$

while in order to prove Lemma 3.16, it suffices to show that

$$
\lim _{n \rightarrow \infty} \sum_{z \in L_{\left\lfloor n^{\alpha_{1}}\right\rfloor} \backslash l_{n}} P_{z}\left(S_{\bar{\tau}_{A}}=x\right)=0 .
$$

Apply reversibility of simple random walk on each $z \in L_{\left\lfloor n^{\alpha_{1}}\right\rfloor} \backslash l_{n}$, we have

$$
\begin{aligned}
\sum_{z \in L_{\left\lfloor n^{\alpha_{1}}\right\rfloor} \backslash l_{n}} P_{z}\left(S_{\bar{\tau}_{A}}=x\right) & =E_{x}\left[\# \text { of visits to } L_{\left\lfloor n^{\alpha_{1}}\right\rfloor} \backslash l_{n} \text { in }\left[0, \bar{\tau}_{A}\right)\right] \\
\leq & \frac{P_{x}\left(\tau_{L_{\left\lfloor n^{\alpha_{1}}\right\rfloor} \backslash l_{n}}<\tau_{L_{0}}\right)}{\min _{z \in L_{\left\lfloor n^{\left.\alpha_{1}\right\rfloor} \backslash l_{n}\right.}} P_{z}\left(\tau_{L_{0}}<\tau_{L_{\left\lfloor n^{\alpha_{1}}\right\rfloor} \backslash l_{n}}\right)} .
\end{aligned}
$$

First, for the denominator of (3.61), note that

$$
\tau_{L_{\left\lfloor n^{\alpha_{1}}\right\rfloor}} \leq \tau_{L_{\left\lfloor n^{\alpha_{1}}\right\rfloor} \backslash l_{n}}
$$


We have for any $z \in L_{\left\lfloor n^{\alpha_{1}}\right\rfloor} \backslash l_{n}$,

$$
P_{z}\left(\tau_{L_{0}}<\tau_{L_{\left\lfloor n^{\alpha_{1}}\right\rfloor} \backslash l_{n}}\right) \geq P_{z}\left(\tau_{L_{0}}<\tau_{l_{\left\lfloor n^{\alpha_{1}}\right\rfloor}}\right) \geq \frac{c}{\left\lfloor n^{\left.\alpha_{1}\right\rfloor}\right.} .
$$

On the other hand, using exactly the same argument as in the proof of Lemma 3.15,

$$
P_{x}\left(\tau_{L_{\left\lfloor n^{\alpha_{1}}\right\rfloor} \backslash l_{n}}<\tau_{L_{0}}\right) \leq \frac{C}{\left\lfloor n^{\alpha_{2}}\right\rfloor} .
$$

Thus, combining (3.61)-(3.63), the proof Lemma 3.16 is complete.

Now with Lemma 3.16, it suffices to prove that

$$
\lim _{n \rightarrow \infty} \sum_{y \in l_{n}}\left[P_{y}\left(S_{\bar{\tau}_{A_{n}}}=x\right)-P_{y}\left(S_{\bar{\tau}_{A}}=x\right)\right]=0 .
$$

Again by reversibility,

$$
P_{y}\left(S_{\bar{\tau}_{A_{n}}}=x\right)=E_{x}\left[\# \text { of visits to } y \text { in }\left[0, \tau_{A_{n}}\right)\right]
$$

and

$$
P_{y}\left(S_{\bar{\tau}_{A}}=x\right)=E_{x}\left[\# \text { of visits to } y \text { in }\left[0, \tau_{A}\right)\right]
$$

which implies that for each $y$

$$
P_{y}\left(S_{\bar{\tau}_{A_{n}}}=x\right)-P_{y}\left(S_{\bar{\tau}_{A}}=x\right)=E_{x}\left[\# \text { of visits to } y \text { in }\left[\tau_{A}, \tau_{A_{n}}\right)\right]
$$

and that

$$
\begin{aligned}
& \sum_{y \in l_{n}}\left[P_{y}\left(S_{\bar{\tau}_{A_{n}}}=x\right)-P_{y}\left(S_{\bar{\tau}_{A}}=x\right)\right] \\
& =E_{x}\left[\# \text { of visits to } l_{n} \text { in }\left[\tau_{A}, \tau_{A_{n}}\right)\right] .
\end{aligned}
$$

Here we use the natural convention that the number of visits equals to 0 over an empty interval. Moreover, define $\bar{T}_{n}=\{-n, n\} \times[0, \infty)$ and

$$
\Gamma_{4}=\inf \left\{n>\tau_{A}, S_{n} \in \bar{T}_{n}\right\} .
$$

Noting that

$$
\left\{\tau_{A}<\Gamma_{4}<\tau_{A_{n}}\right\} \subset\left\{\tau_{A}<\tau_{A_{n}}\right\} \subset\left\{\tau_{\bar{T}_{n}}<\tau_{A_{n}}\right\},
$$

thus by strong Markov property, one can see that

$$
E_{x}\left[\# \text { of visits to } l_{n} \text { in }\left[\tau_{A}, \tau_{A_{n}}\right)\right] \leq \frac{P_{x}\left(\tau_{\bar{T}_{n}}<\tau_{A_{n}}\right)}{\min _{z \in l_{n}} P_{z}\left(\tau_{A_{n}}<\tau_{l_{n}}\right)} .
$$

First, for any $z=\left(z^{(1)}, z^{(2)}\right) \in l_{n}$, consider

$$
\left(z^{(1)}, 0\right)+\left[-\left\lfloor n^{\alpha_{1}}\right\rfloor,\left\lfloor n^{\alpha_{1}}\right\rfloor\right] \times\left[0,\left\lfloor n^{\alpha_{1}}\right\rfloor\right]
$$

By Lemma 2.4 and translation/reflection invariance of simple random walk,

$$
\begin{aligned}
P_{z}\left(\tau_{A_{n}}<\tau_{l_{n}}\right) & \geq P_{0}\left(\tau_{\partial_{u}^{i n} I_{\left\lfloor n^{\left.\alpha_{1}\right\rfloor}\right.}}<\tau_{L_{0}}\right) \\
& \geq P_{0}\left(\tau_{\partial_{u}^{i n} I_{\left\lfloor n^{\left.\alpha_{1}\right\rfloor}\right.}}=\tau_{\partial^{i n} I_{\left\lfloor n^{\left.\alpha_{1}\right\rfloor}\right.}}\right) \\
& \geq \frac{1}{2} P_{0}\left(\tau_{\partial^{i n} I_{\left\lfloor n^{\alpha_{1}}\right\rfloor}}<\tau_{L_{0}}\right) \\
& \geq \frac{1}{2} P_{0}\left(\tau_{L_{\left\lfloor n^{\alpha_{1}}\right\rfloor}}<\tau_{L_{0}}\right)=\frac{1}{8\left\lfloor n^{\left.\alpha_{1}\right\rfloor}\right.} .
\end{aligned}
$$


On the other hand, we have

$$
\begin{aligned}
P_{x}\left(\tau_{\bar{T}_{n}}<\tau_{A_{n}}\right) & \leq P_{x}\left(\tau_{\bar{T}_{n}}<\tau_{L_{0}}\right) \\
& \leq C P_{0}\left(\tau_{\partial^{i n} I_{\lfloor n / 2\rfloor}}<\tau_{L_{0}}\right) \\
& \leq 2 C P_{0}\left(\tau_{\partial_{u}^{i n} I_{\lfloor n} \alpha_{1\rfloor}}=\tau_{\partial^{i n} I_{\lfloor n / 2\rfloor}}\right) \leq 2 C P_{0}\left(\tau_{L_{\lfloor n / 2\rfloor}}<\tau_{L_{0}}\right) \leq \frac{C}{n} .
\end{aligned}
$$

Now combining (3.65)-(3.68), we have shown (3.64) and the proof of Proposition 3.7 is complete.

3.8. Proof of Proposition 3.8. At this point, in order to prove Theorem 1.3, we only need to show that for all sufficiently large $n$ and any $y \in l_{n}, 2 \mathcal{H}_{B o x(n)}(y) / \mathcal{H}_{D_{n}}(0)$ can be arbitrarily close to one. First, for any $y \in l_{n}$, define

$$
M(y, n)=n+\left|y^{(1)}\right|, \quad m(y, n)=n-\left|y^{(1)}\right| .
$$

Recall that $\operatorname{Box}(n)=[-n, n] \times\left[0,\left\lfloor n^{\alpha_{1}}\right\rfloor\right]$ and that $l_{n}=\left[-\left\lfloor n^{\alpha_{2}}\right\rfloor,\left\lfloor n^{\alpha_{2}}\right\rfloor\right] \times\left\{\left\lfloor n^{\alpha_{1}}\right\rfloor\right\}$. We have

$$
n-\left\lfloor n^{\alpha_{2}}\right\rfloor \leq m(y, n) \leq n \leq M(y, n) \leq n+\left\lfloor n^{\alpha_{2}}\right\rfloor .
$$

Moreover, noting that

$$
\operatorname{Box}(n) \subset\left[y^{(1)}-M(y, n), y^{(1)}+M(y, n)\right] \times\left[0,\left\lfloor n^{\alpha_{1}}\right\rfloor\right]
$$

and that

$$
\left[y^{(1)}-m(y, n), y^{(1)}+m(y, n)\right] \times\left[0,\left\lfloor n^{\alpha_{1}}\right\rfloor\right] \subset \operatorname{Box}(n),
$$

by definition we have

$$
H_{\left[y^{(1)}-M(y, n), y^{(1)}+M(y, n)\right] \times\left[0,\left\lfloor n^{\alpha_{1}}\right\rfloor\right]}(y) \leq \mathcal{H}_{B o x(n)}(y)
$$

and

$$
H_{\left[y^{(1)}-m(y, n), y^{(1)}+m(y, n)\right] \times\left[0,\left\lfloor n^{\alpha_{1}}\right\rfloor\right]}(y) \geq \mathcal{H}_{B o x(n)}(y) .
$$

Thus, combine translation invariance and Theorem 3.1, and note that for all $y \in l_{n}, M^{-1}(y, n)-$ $n^{-1}=o\left(n^{-1}\right), m^{-1}(y, n)-n^{-1}=o\left(n^{-1}\right)$. It is immediate to see that Proposition 3.8 is equivalent to the following statement:

Lemma 3.17. For all integers $m, n>0$, define

$$
\widehat{\operatorname{Box}}(m, n)=[-n, n] \times[-m, 0] .
$$

For any $\epsilon>0$, we have

$$
\mathcal{H}_{D_{n}}(0)-2 \mathcal{H}_{\widehat{\operatorname{Box}}(m, n)}(0) \in\left[0, \frac{\epsilon}{n}\right)
$$

for all sufficiently large $n$ and all $0<m \leq 2 n^{\alpha_{1}}$.

Proof: First, for the lower bound estimate, note that

$$
D_{n} \subset \widehat{\operatorname{Box}}(m, n)
$$

and that by the definition of harmonic measure

$$
\mathcal{H}_{D_{n}}(0)=\lim _{k \rightarrow \infty} P_{(k, 0)}\left(\tau_{D_{n}}=\tau_{0}\right)
$$

and that

$$
\mathcal{H}_{\widehat{B O x}(m, n)}(0)=\lim _{k \rightarrow \infty} P_{(k, 0)}\left(\tau_{\widehat{B o x}(m, n)}=\tau_{0}\right) .
$$

Moreover, by symmetry we have for all $k>n$,

$$
P_{(k, 0)}\left(\tau_{D_{n}}=\tau_{0}\right)=2 P_{(k, 0)}\left(\tau_{D_{n}}=\tau_{0}, S_{\tau_{0}-1}=(0,1)\right) .
$$


At the same time one can see that in the event $\left\{\tau_{\widehat{B o x}(m, n)}=\tau_{0}\right\}$, the random walk has to visit 0 through $(0,1)$, which implies that

$$
P_{(k, 0)}\left(\tau_{D_{n}}=\tau_{0}, S_{\tau_{0}-1}=(0,1)\right) \geq P_{(k, 0)}\left(\tau_{\widehat{B o x}(m, n)}=\tau_{0}\right) .
$$

Taking the limit as $k \rightarrow \infty$, we have shown the lower bound estimate. For the upper bound estimate, again we note that for each sufficiently large $k$ and a random walk starting from $(k, 0)$,

$$
\begin{aligned}
& \left\{\tau_{D_{n}}=\tau_{0}, S_{\tau_{0}-1}=(0,1)\right\} \backslash\left\{\tau_{\widehat{B o x}(m, n)}=\tau_{0}\right\} \\
& =\left\{\tau_{D_{n}}=\tau_{0}, S_{\tau_{0}-1}=(0,1)\right\} \cap\left\{\tau_{\widehat{B o x}(m, n) \backslash D_{n}}<\tau_{D_{n}}\right\},
\end{aligned}
$$

which, by strong Markov property, implies that

$$
\begin{aligned}
& P_{(k, 0)}\left(\tau_{D_{n}}=\tau_{0}, S_{\tau_{0}-1}=(0,1)\right)-P_{(k, 0)}\left(\tau_{\widehat{B o x}(m, n)}=\tau_{0}\right) \\
\leq & \max _{y \in \widehat{\operatorname{Box}}(m, n) \backslash D_{n}} P_{y}\left(\tau_{(0,1)}<\tau_{D_{n}}\right) .
\end{aligned}
$$

Now in order to find the upper bound of the right hand side of (3.71), we consider the following two cases based on the location of point $y=\left(y^{(1)}, y^{(2)}\right) \in \widehat{\operatorname{Box}}(m, n) \backslash D_{n}$ :

\section{Case 1:}

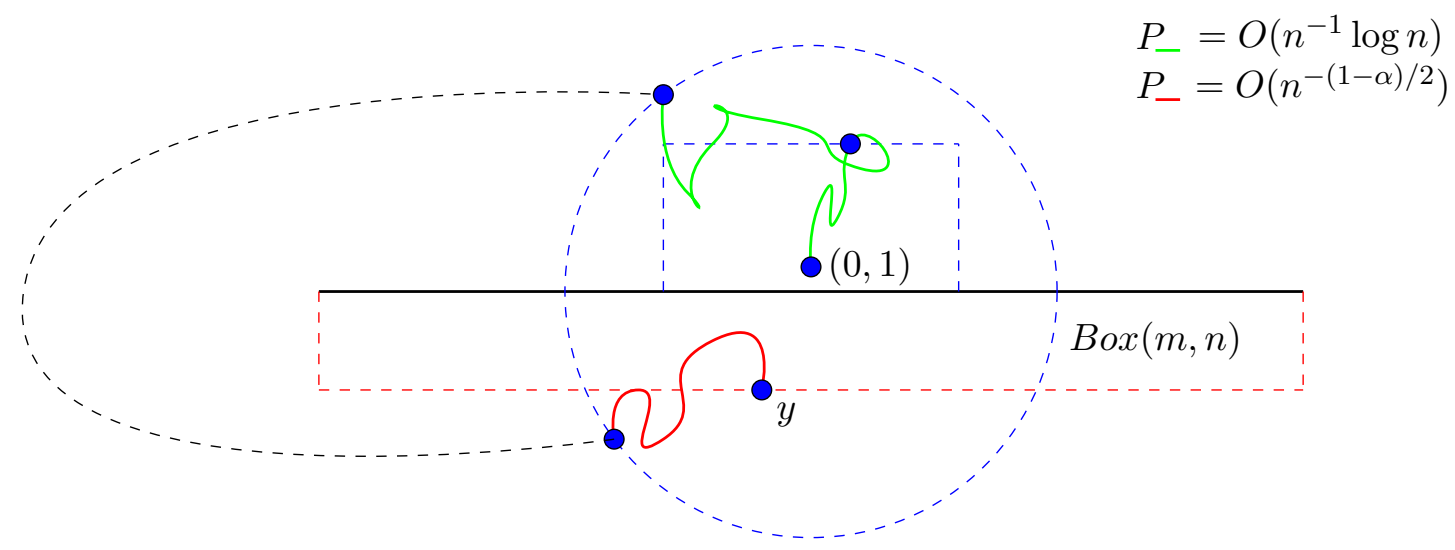

FiguRE 3.3. Illustration of proof for Case 1

If $\left|y^{(1)}\right| \leq n / 3$, for all nearest neighbor paths starting at $y$ which hit $(0,1)$ before $D_{n}$, they first have to hit $\partial^{\text {out }} B(0, n / 2)$. Thus, we have

$$
\begin{aligned}
P_{y}\left(\tau_{(0,1)}<\tau_{D_{n}}\right) & =\sum_{z \in \partial^{\text {out }} B(0, n / 2)} P_{y}\left(\tau_{n / 2}<\tau_{D_{n}}, S_{\tau_{n / 2}}=z\right) P_{z}\left(\tau_{(0,1)}<\tau_{D_{n}}\right) \\
& \leq P_{y}\left(\tau_{n / 2}<\tau_{D_{n}}\right) \max _{z \in \partial^{\text {out }} B(0, n / 2)} P_{z}\left(\tau_{(0,1)}<\bar{\tau}_{D_{n}}\right) .
\end{aligned}
$$

For the first term of the right hand side of (3.72), recalling that $d\left(y, D_{n}\right)=\left|y^{(2)}\right|=m \leq 2 n^{\alpha_{1}}$ and that $\left|y^{(1)}\right|<n / 3$, by the same Beurling estimate, there exists a constant $C<\infty$ independent to the choices of $n, m$ and $y$ satisfying Case 1 such that

$$
P_{y}\left(\tau_{n / 2}<\tau_{D_{n}}\right) \leq C n^{-\left(1-\alpha_{1}\right) / 2} .
$$


At the same time, for any $z \in \partial^{o u t} B(0, n / 2)$, to control the upper bound on $P_{z}\left(\tau_{(0,1)}<\bar{\tau}_{D_{n}}\right)$, one can concentrate on the upper half plane, since each path from $y$ to $(0,1)$ must pass through some point $z \in \partial^{\text {out }} B(0, n / 2) \cap\left\{x \in \mathbb{H}: x^{(2)}>0\right\}$. Now for any such $z$, by reversibility, we have

$$
P_{z}\left(\tau_{(0,1)}<\bar{\tau}_{D_{n}}\right)=E_{(0,1)}\left[\# \text { of visits to } z \text { in }\left[0, \tau_{D_{n} \cup\{(0,1)\}}\right)\right] \leq \frac{P_{(0,1)}\left(\tau_{z}<\bar{\tau}_{D_{n}}\right)}{P_{z}\left(\bar{\tau}_{D_{n}}<\tau_{z}\right)}
$$

For the numerator, note that for all sufficiently large $n,[-\lfloor n / 3\rfloor,\lfloor n / 3\rfloor] \times[0,\lfloor n / 3\rfloor] \subset B(0, n / 2)$. Applying the same argument as we repeatedly used in this paper, we have

$$
P_{(0,1)}\left(\tau_{z}<\bar{\tau}_{D_{n}}\right) \leq \frac{C}{n} .
$$

At the same time,

$$
\begin{aligned}
& P_{z}\left(\bar{\tau}_{D_{n}}<\tau_{z}\right) \\
& \quad \geq \sum_{\substack{\text { out } B\left(z, \frac{z^{(2)}}{2}\right) \\
P_{z}}}\left(\bar{\tau}_{\partial^{\text {out }} B\left(z, \frac{z(2)}{2}\right)}<\tau_{z}, \bar{\tau}_{\partial^{\text {out }} B\left(z, \frac{z^{(2)}}{2}\right)}=\tau_{w}\right) P_{w}\left(\bar{\tau}_{D_{n}}<\tau_{\partial^{\text {out }} B\left(z, \frac{z^{(2)}}{3}\right)}\right) .
\end{aligned}
$$

And by invariance principle and the fact that $z^{(2)} \in(0, n]$, there is a constant $c>0$ independent to the choices of $n, z$, and $w$ such that

$$
P_{w}\left(\bar{\tau}_{D_{n}}<\tau_{\partial^{\text {out }} B\left(z, \frac{z(2)}{3}\right)}\right) \geq c .
$$

Thus by Lemma 3.14,

$$
P_{z}\left(\bar{\tau}_{D_{n}}<\tau_{z}\right) \geq c P_{z}\left(\bar{\tau}_{\partial^{\text {out }} B\left(z, \frac{z^{(2)}}{2}\right)}<\tau_{z}\right) \geq \frac{c}{\left(\log \frac{z^{(2)}}{2}\right)^{2}} \geq \frac{c}{(\log n)^{2}}
$$

which by (3.74) implies that

$$
P_{z}\left(\tau_{(0,1)}<\bar{\tau}_{D_{n}}\right) \leq \frac{C(\log n)^{2}}{n} .
$$

Now combining (3.71), (3.72), (3.73), and (3.75),

$$
P_{y}\left(\tau_{(0,1)}<\tau_{D_{n}}\right) \leq C n^{-\left(3-\alpha_{1}\right) / 2}(\log n)^{2} \ll n^{-1}
$$

and thus our lemma holds when $y$ in Case 1.

\section{Case 2:}

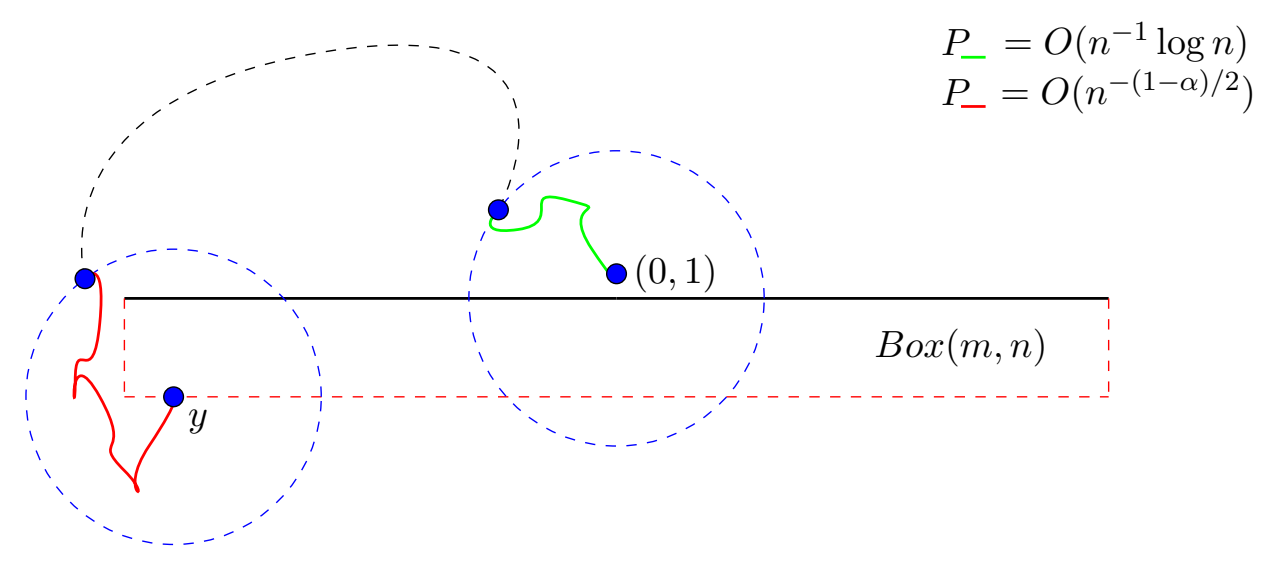

Figure 3.4. Illustration of proof for Case 2 
Otherwise, if $\left|y^{(1)}\right|>n / 3$, our proof follows the same techniques on slightly different stopping times. Consider two neighborhoods: $B\left(0, \frac{n}{7}\right)$ and $B\left(y, \frac{n}{7}\right)$. It is easy to see that

$$
\partial^{\text {out }} B\left(0, \frac{n}{7}\right) \cap \partial^{\text {out }} B\left(y, \frac{n}{7}\right)=\emptyset \text {. }
$$

Using the same argument as in Case 1,

$$
P_{y}\left(\tau_{(0,1)}<\tau_{D_{n}}\right)=\sum_{w \in \partial^{\text {out }} B\left(y, \frac{n}{7}\right)} P_{y}\left(\tau_{\partial^{\text {out }} B\left(y, \frac{n}{7}\right)}<\tau_{D_{n}}, S_{\tau_{\partial^{o u t}} B\left(y, \frac{n}{7}\right)}=w\right) P_{w}\left(\tau_{(0,1)}<\tau_{D_{n}}\right) .
$$

Moreover, for any $w \in \partial^{\text {out }} B\left(y, \frac{n}{7}\right)$ the random walk starting at $w$ has to first visit $\partial^{\text {out }} B\left(0, \frac{n}{7}\right)$ before ever reaches $(0,1)$. This implies that

$$
\begin{aligned}
P_{w}\left(\tau_{(0,1)}<\tau_{D_{n}}\right) & =\sum_{z \in \partial^{\text {out }} B\left(0, \frac{n}{7}\right)} P_{w}\left(\tau_{n / 7}<\tau_{D_{n}}, S_{\tau_{n / 7}}=z\right) P_{z}\left(\tau_{(0,1)}<\tau_{D_{n}}\right) \\
& \leq \max _{z \in \partial^{\text {out }} B\left(0, \frac{n}{7}\right)} P_{z}\left(\tau_{(0,1)}<\tau_{D_{n}}\right) .
\end{aligned}
$$

Thus, we have

$$
P_{y}\left(\tau_{(0,1)}<\tau_{D_{n}}\right) \leq P_{y}\left(\tau_{\partial^{\text {out }} B\left(y, \frac{n}{7}\right)}<\tau_{D_{n}}\right) \max _{z \in \partial^{\text {out }} B\left(0, \frac{n}{7}\right)} P_{z}\left(\tau_{(0,1)}<\tau_{D_{n}}\right) .
$$

Now since $y^{(2)}=-m \geq-2 n^{\alpha_{1}}$, it is easy to see that

$$
\operatorname{rad}\left(B\left(y, \frac{n}{7}\right) \cap D_{n}\right) \geq \frac{n}{4}
$$

for all sufficiently large $n$. Thus by (3.73) and (3.75), there exists a constant $C<\infty$ independent to the choices of $n, m$ and $y$ satisfying Case 2 such that

$$
P_{y}\left(\tau_{\partial^{\text {out }} B\left(y, \frac{n}{7}\right)}<\tau_{D_{n}}\right) \leq C n^{-\left(1-\alpha_{1}\right) / 2} .
$$

and that

$$
\max _{z \in \partial^{\text {out }} B\left(0, \frac{n}{7}\right)} P_{z}\left(\tau_{(0,1)}<\tau_{D_{n}}\right) \leq \frac{C(\log n)^{2}}{n} .
$$

Therefore, we also have

$$
P_{y}\left(\tau_{(0,1)}<\tau_{D_{n}}\right) \leq C n^{-\left(3-\alpha_{1}\right) / 2}(\log n)^{2} \ll n^{-1},
$$

and thus our lemma holds when $y$ in Case 2, and the proof of Lemma 3.17 is complete.

With Lemma 3.17, we have concluded the proof of Proposition 3.8.

\section{References}

Antunović, T. and Procaccia, E. B. Stationary Eden model on Cayley graphs. Ann. Appl. Probab., 27 (1), 517-549 (2017). MR3619794.

Berger, N., Kagan, J. J., and Procaccia, E. B. Stretched IDLA. ALEA Lat. Am. J. Probab. Math. Stat., 11 (1), 471-481 (2014). MR3274641.

Delmotte, T. Parabolic Harnack inequality and estimates of Markov chains on graphs. Rev. Mat. Iberoamericana, 15 (1), 181-232 (1999). MR1681641.

Kesten, H. Hitting probabilities of random walks on $\mathbf{Z}^{d}$. Stochastic Process. Appl., 25 (2), 165-184 (1987). MR915132.

Lawler, G. F. and Limic, V. The Beurling estimate for a class of random walks. Electron. J. Probab., 9, no. 27, 846-861 (2004). MR2110020. 
Lawler, G. F. and Limic, V. Random walk: a modern introduction, volume 123 of Cambridge Studies in Advanced Mathematics. Cambridge University Press, Cambridge (2010). ISBN 978-0521-51918-2. MR2677157.

$\mathrm{Mu}$, Y., Procaccia, E. B., and Zhang, Y. Scaling limit of DLA on a long line segment. ArXiv Mathematics e-prints (2019). arXiv: 1912.02370.

Procaccia, E. B., Ye, J., and Zhang, Y. Stationary DLA is well defined. J. Stat. Phys., 181 (4), 1089-1111 (2020). MR4163494.

Procaccia, E. B. and Zhang, Y. Stationary harmonic measure and DLA in the upper half plane. $J$. Stat. Phys., 176 (4), 946-980 (2019). MR3990220.

Procaccia, E. B. and Zhang, Y. On sets of zero stationary harmonic measure. Stochastic Process. Appl., 131, 236-252 (2021). MR4153884. 